\title{
CONVERGENCE OF A NUMERICAL SCHEME FOR A MIXED HYPERBOLIC-PARABOLIC SYSTEM IN TWO SPACE DIMENSIONS *
}

\author{
Elena Rossi ${ }^{1}$ and Veronika SchlePeR ${ }^{2}$
}

\begin{abstract}
We prove the convergence of an explicit numerical scheme for the discretization of a coupled hyperbolic-parabolic system in two space dimensions. The hyperbolic part is solved by a Lax-Friedrichs method with dimensional splitting, while the parabolic part is approximated by an explicit finitedifference method. For both equations, the source terms are treated by operator splitting. To prove convergence of the scheme, we show strong convergence of the hyperbolic variable, while convergence of the parabolic part is obtained only weakly* in $\mathbf{L}^{\infty}$. The proof relies on the fact that the hyperbolic flux depends on the parabolic variable through a convolution function. The paper also includes numerical examples that document the theoretically proved convergence and display the characteristic behaviour of the Lotka-Volterra equations.
\end{abstract}

Mathematics Subject Classification. 65M12, 35M30.

Received November 20, 2014. Revised July 1, 2015.

Published online March 2, 2016.

\section{INTRODUCTION}

We consider the following Cauchy problem in two space dimensions:

$$
\begin{aligned}
& \partial_{t} u+\operatorname{div}(f(u) \boldsymbol{v}(w))=(\alpha w-\beta) u \\
& \partial_{t} w-\mu \Delta w=(\gamma-\delta u) w \\
& u(0, x, y)=u_{o}(x, y) \\
& w(0, x, y)=w_{o}(x, y) .
\end{aligned}
$$

This is a generalization of the predator-prey model presented in [8]. In particular, $u=u(t, x, y)$ and $w=$ $w(t, x, y)$ represent respectively the predator and the prey densities at time $t \in \mathbb{R}^{+}$and position $(x, y) \in \mathbb{R}^{2}$. The parameters $\alpha, \beta, \gamma, \delta$ appearing in system (1.1) are all positive, $\mu$ is strictly positive. More precisely, $\alpha$ is

\footnotetext{
Keywords and phrases. Numerical analysis, mixed systems of partial differential equations, coupled equations, Lax-Friedrichs method, finite difference schemes, nonlocal conservation laws.

* The first author is member of the Gruppo Nazionale per l'Analisi Matematica, la Probabilità e le loro Applicazioni (GNAMPA) of the Istituto Nazionale di Alta Matematica (INdAM). The second author would like to thank the German Research Foundation (DFG) for financial support of the project within the Cluster of Excellence in Simulation Technology (EXC 310/2) at the University of Stuttgart.

1 University of Milano-Bicocca, Via Cozzi 55, 20125 Milano, Italy. e.rossi50@campus.unimib.it

2 University of Stuttgart, Pfaffenwaldring 57, 70569 Stuttgart, Germany. veronika.schleper@mathematik.uni-stuttgart.de
} 
the predator birth rate due to feeding on prey, $\beta$ is the predator mortality rate, $\gamma$ is the prey birth rate, $\delta$ is the prey mortality rate due to predators and $\mu$ represents the diffusion speed of prey.

Predator-prey models are widely studied in the literature since a long time, starting with the pioneering works of Lotka [15] and Volterra [17] in the 1920s. The model proposed therein is based on ordinary differential equations modeling the interactions of two species' populations $u$ (predators) and $w$ (prey), where birth and death rates depend on the interactions, see equation (1.2).

$$
\frac{\mathrm{d}}{\mathrm{d} t} u=(\alpha w-\beta) u, \quad \frac{\mathrm{d}}{\mathrm{d} t} w=(\gamma-\delta u) w .
$$

This basic model was extended subsequently to obtain more refined predictions of population sizes $[3,4,13]$ or to model the immune system response to infectious diseases $[5,16]$. Further applications of the Lotka-Volterra model (1.2) and variations of it can be found in economy, see e.g. [12] for a pioneering work.

All these models are based on ordinary differential equations, thus implicitly assuming a homogeneous distribution of the species in space. Model (1.1) overcomes this deficiency and allows for spacial variations of predators and prey. More precisely, we assume that prey diffuse in the whole space without preferred direction of motion, while predators are attracted by prey in a certain radius around them. To model this finite-range non-local behavior, the velocity vector $\boldsymbol{v}$ of the predators depends on a convolution of the prey density with a kernel function measuring e.g. the ability of the predators to feel the presence of prey at a certain distance. This non local term in the flux of (1.1a) causes the predators to move in direction of the highest prey density, thus chasing the prey. Note that the prey does not try to escape the predators.

In [8], the well-posedness of (1.1) was shown for $f(u)=u$ and initial data in $\mathbf{L}^{1} \cap \mathbf{L}^{\infty}\left(\mathbb{R}^{n} ; \mathbb{R}\right)$ with the additional constraint that $u_{\mathrm{o}}$ is of bounded total variation. The topic of the present work is to study the convergence of a finite difference scheme for the mixed hyperbolic-parabolic system (1.1). We choose a Lax-Friedrichs type method for the hyperbolic part, including a modification to deal with the non local term $\boldsymbol{v}$, and a standard five-point stencil for the discretization of the parabolic part, see also (2.4) in Section 2. In particular, we consider a different diffusion constant in the Lax-Friedrichs flux, see (2.4d) and Remark 2.4. This is done in order to balance the contributions of the space-time dependent velocity field $\boldsymbol{v}$ to obtain positivity and an $\mathbf{L}^{\infty}$ bound for $u$.

Since the velocity function $\boldsymbol{v}$ depends only on $w$, we could view equation (1.1a) as a standard hyperbolic equation with space and time dependent flux function $\tilde{\boldsymbol{f}}(t, x, u):=f(u) \boldsymbol{v}(t, x)$. Equations of such type have been widely studied in the literature and especially the convergence of finite volume schemes is established in $[6,7,11,14]$ under different assumptions on $\boldsymbol{v}(t, x)$. Recently, a Lax-Friedrichs type method for a nonlocal hyperbolic conservation law was studied in $[1,2]$. Due to the coupling of $(1.1 \mathrm{a})$ and $(1.1 \mathrm{~b})$ through the velocity function $\boldsymbol{v}$ and the source terms, the above results do not apply to the present case. The same holds true for the well known standard convergence results for finite difference discretizations of (quasi)linear parabolic equations, since these results are usually based on estimates in the discrete $l^{2}$-norm. Here, the coupling of the parabolic part to a hyperbolic equation forces us to study the finite difference scheme in an $l^{1}$ setting.

To prove the convergence of the numerical scheme below, we make the following assumptions on the functions $f$ and $\boldsymbol{v}$ appearing in (1.1):

$(\mathbf{f}): f \in \mathbf{C}^{2}(\mathbb{R} ; \mathbb{R})$ and $f(0)=0$;

$(\mathbf{v}): v:\left(\mathbf{L}^{1} \cap \mathbf{L}^{\infty}\right)\left(\mathbb{R}^{2} ; \mathbb{R}\right) \rightarrow\left(\mathbf{C}^{2} \cap \mathbf{W}^{2, \infty}\right)\left(\mathbb{R}^{2} ; \mathbb{R}^{2}\right)$ depends on $w$ through a convolution in space, i.e. $\boldsymbol{v}(w):=$ $\boldsymbol{v}(\eta * w)$ for a space dependent convolution kernel $\eta \in \mathbf{L}^{1}\left(\mathbb{R}^{2} ; \mathbb{R}\right)$. Furthermore, there exist a constant $K$ and an increasing map $C \in \mathbf{L}_{\text {loc }}^{\infty}\left(\mathbb{R}^{+} ; \mathbb{R}^{+}\right)$such that for all $w \in\left(\mathbf{L}^{1} \cap \mathbf{L}^{\infty}\right)\left(\mathbb{R}^{2} ; \mathbb{R}\right)$

$$
\begin{aligned}
\|\nabla \boldsymbol{v}(w)\|_{\mathbf{L}^{\infty}\left(\mathbb{R}^{2} ; \mathbb{R}^{2 \times 2}\right)} & \leq K\|w\|_{\mathbf{L}^{\infty}\left(\mathbb{R}^{2} ; \mathbb{R}\right)} \\
\|\nabla \boldsymbol{v}(w)\|_{\mathbf{L}^{1}\left(\mathbb{R}^{2} ; \mathbb{R}^{2 \times 2}\right)} & \leq K\|w\|_{\mathbf{L}^{1}\left(\mathbb{R}^{2} ; \mathbb{R}\right)} \\
\|\nabla(\nabla \cdot \boldsymbol{v}(w))\|_{\mathbf{L}^{\infty}\left(\mathbb{R}^{2} ; \mathbb{R}^{2}\right)} & \leq C\left(\|w\|_{\mathbf{L}^{\infty}\left(\mathbb{R}^{2} ; \mathbb{R}\right)}\right) .
\end{aligned}
$$


With slight abuse of notation, we will also write $\boldsymbol{v}(t, x, y)$ instead of $\boldsymbol{v}(w)(t, x, y)$, to improve readability. Note that the case $f(u)=u$ is the one considered in [8]. An example of a function $\boldsymbol{v}$ that fulfills all requirements of assumption ( $\mathbf{v})$ above can be found in Section 5. The initial data $\left(u_{o}, w_{o}\right)$ are chosen to fulfill the assumption

(0): $\left(u_{o}, w_{o}\right) \in\left(\mathbf{L}^{1} \cap \mathbf{L}^{\infty} \cap \mathbf{B V}\right)\left(\mathbb{R}^{2} ; \mathbb{R}^{+}\right) \times\left(\mathbf{L}^{1} \cap \mathbf{L}^{\infty} \cap \mathbf{B V}\right)\left(\mathbb{R}^{2} ; \mathbb{R}^{+}\right)$are positive-valued functions, i.e. $u_{o} \geq 0$ and $w_{o} \geq 0$ for a.e. $(x, y) \in \mathbb{R}^{2}$.

Remark 1.1. Under the assumption (f), existence and uniqueness of the solution to (1.1) in the space $\left(\mathbf{L}^{1} \cap \mathbf{L}^{\infty} \cap \mathbf{B V}\right)\left(\mathbb{R}^{n} ; \mathbb{R}^{+}\right) \times\left(\mathbf{L}^{1} \cap \mathbf{L}^{\infty}\right)\left(\mathbb{R}^{n} ; \mathbb{R}^{+}\right)$follow by a straightforward extension of the results of [8].

The paper is organized as follows. In Section 2, we introduce basic notations and describe the algorithm in details. To prove convergence of the given scheme, we derive bounds on the variables $u$ and $w$ in various norms in Section 3 that are needed to conclude the convergence of the scheme in Section 4. Finally, Section 5 is devoted to numerical examples including experimental convergence studies.

\section{The Algorithm}

We introduce a uniform mesh of width $h$ along both $x$ and $y$ axes, given by the cartesian grid whose points are of the form

$$
\left\{\left(x_{i}, y_{i}\right) \mid x_{i}=i h, y_{j}=j h, i, j \in \mathbb{Z}\right\} .
$$

With slight abuse of notation, we will also write $x_{i, j}:=\left(x_{i}, y_{j}\right), x_{i+1 / 2, j}:=\left(x_{i+1 / 2}, y_{j}\right)=((i+1 / 2) h, j h)$ and $x_{i, j+1 / 2}$ defined analogously. Furthermore, we define the parabolic time step $\tau_{p}=\frac{h^{2}}{4 \mu}$ and let the time step $\tau$ be such that

$$
\tau=\tau_{p} \max \left\{n \in \mathbb{N}: \frac{n \tau_{p}}{h}\left\|\partial_{u} f\right\|_{\mathbf{L}^{\infty}}\|\boldsymbol{v}\|_{\mathbf{L}^{\infty}}<\frac{1}{4}\right\}=: m \tau_{p} .
$$

In other words, $\tau$ is a multiple of $\tau_{p}$ that satisfies the following CFL condition:

$$
\frac{\tau}{h}\left\|\partial_{u} f\right\|_{\mathbf{L} \infty}\|\boldsymbol{v}\|_{\mathbf{L}^{\infty}}<\frac{1}{4}
$$

We also define $\lambda=\frac{\tau}{h}$. We remark that $\|\boldsymbol{v}\|_{\mathbf{L}} \infty$ is assumed to be the global supremum of $\boldsymbol{v}$ throughout the paper to simplify notations and estimates. This assumption can however be relaxed to a time-step-wise supremum, thus leading to an adaptive time stepping strategy. The same holds for $\left\|\partial_{u} f\right\|_{\mathbf{L}^{\infty}}$. Note further that $\tau$ is fixed by the choice of $\tau_{p}$, contrarily to the standard case for hyperbolic equations, where (2.1) provides some freedom in the choice of $\tau$. Finally, let us remark that the time step $\tau_{p}$ is chosen such that the numerical approximation of $w^{n}$ has a particularly simple form (see $(2.4 \mathrm{~b})$ below). This will improve the readability of the estimates in the following sections. However, any time step $\tau_{p}$ that yields a stable discretization of $w^{n}$ will also lead to a convergent scheme.

Let $(u(t, x, y), w(t, x, y))$ be the unique solution to (1.1) (see [8] for an existence and uniqueness result in the case of $f(u)=u$ and Remark 1.1 for the general case, under assumption (f)). To compute the solution numerically we set

$$
u_{i, j}^{o}=\frac{1}{h^{2}} \int_{I_{i, j}} u_{o}(x, y) \mathrm{d} x \mathrm{~d} y, \quad w_{i, j}^{o}=\frac{1}{h^{2}} \int_{I_{i, j}} w_{o}(x, y) \mathrm{d} x \mathrm{~d} y
$$

where

$$
I_{i, j}=\left[\left(i-\frac{1}{2}\right) h,\left(i+\frac{1}{2}\right) h\right] \times\left[\left(j-\frac{1}{2}\right) h,\left(j+\frac{1}{2}\right) h\right]
$$


so that $u_{i, j}^{o}$ and $w_{i, j}^{o}$ are the cell averages of $u_{o}(x, y)$ and $w_{o}(x, y)$ respectively over the $(i, j)$-th cell. By $(2.2)$ it follows easily that

$$
\begin{aligned}
\left\|u^{o}\right\|_{\mathbf{L}^{\infty}} & \leq\left\|u_{o}\right\|_{\mathbf{L}^{\infty}} & \left\|w^{o}\right\|_{\mathbf{L}^{\infty}} & \leq\left\|w_{o}\right\|_{\mathbf{L}^{\infty}} \\
\left\|u^{o}\right\|_{\mathbf{L}^{1}} & \leq\left\|u_{o}\right\|_{\mathbf{L}^{1}} & \left\|w^{o}\right\|_{\mathbf{L}^{1}} & \leq\left\|w_{o}\right\|_{\mathbf{L}^{1}} \\
\operatorname{TV}\left(u^{o}\right) & \leq \operatorname{TV}\left(u_{o}\right) & \operatorname{TV}\left(w^{o}\right) & \leq \operatorname{TV}\left(w_{o}\right),
\end{aligned}
$$

see also [9]. For simplicity, we denote $v_{i+1 / 2, j}^{n}=\boldsymbol{v}\left(n \tau, x_{i+1 / 2}, y_{j}\right) \cdot \boldsymbol{n}_{i+1 / 2, j}$, where $\boldsymbol{n}_{i+1 / 2, j}$ is the normal vector of the cell boundary at $x_{i+1 / 2, j}$, pointing from the cell with value $u_{i, j}$ to the cell with value $u_{i+1, j}$. The definition of $v_{i, j+1 / 2}^{n}$ follows analogously. Note that this definition, together with the rectangular grid, implies that $v_{i+1 / 2, j}^{n}$ is the $x$-component of the vector $\boldsymbol{v}^{n}$ at $x_{i+1 / 2, j}$. Analogously, $v_{i, j+1 / 2}^{n}$ denotes the $y$-component.

To approximate (1.1), we use a finite-difference scheme for the parabolic part and a Lax-Friedrichs type finite volume method for the hyperbolic part. In both equations, the source terms are treated by operator splitting, using a second order Runge-Kutta method. The nonlinear coupling of (1.1) is numerically resolved by a sequential coupling of the parabolic and the hyperbolic equation. In other words, we start computing an approximation of $w^{n}$, solving the parabolic equation by an explicit scheme with smaller time step $\tau_{p}$, thus having to perform $m$ small time steps to reach the hyperbolic time step $\tau$ defined in (2.1). This step involves the values of $u$ at the previous time step $t^{n-1}:=(n-1) \tau$ for the discretization of the source term. Once, $w^{n}$ is computed, we can use it to compute the velocity field $\boldsymbol{v}\left(w^{n}\right)$ and the source term in the hyperbolic equation. This balance law is now integrated by means of a Lax-Friedrichs type scheme with dimensional splitting, while its source term is included using a second order Runge-Kutta method, analogously to the parabolic equation. Note that the second order discretization of the source terms is necessary to guarantee the positivity of the approximate solution, as shown in Section 3.1.

Note that, in order to be able to give a valid meaning to $\boldsymbol{v}\left(w^{n}\right)$, involving a convolution, we set $w^{n}:=$ $\sum_{i j} w_{i, j}^{n} \chi_{I_{i, j}}$. The algorithm is now defined as follows:

Algorithm 2.1 (Mixed scheme).

$$
\begin{aligned}
& \text { for } n=0, \ldots N-1 \\
& W^{n, 0}=w^{n} \\
& \text { for } l=0, \ldots, m-1 \\
& W_{i, j}^{n, l+1}=\frac{1}{4}\left(W_{i+1, j}^{n, l}+W_{i-1, j}^{n, l}+W_{i, j+1}^{n, l}+W_{i, j-1}^{n, l}\right) \\
& \times\left[1+\tau_{p}\left(\gamma-\delta u_{i, j}^{n}\right)\left(1+\frac{\tau_{p}}{2}\left(\gamma-\delta u_{i, j}^{n}\right)\right)\right] \\
& w^{n+1}=W^{n, m} \\
& F\left(u_{1}, u_{2}, t, x, y\right)=\frac{1}{2}\left(f\left(u_{1}\right)+f\left(u_{2}\right)\right) v(t, x, y)-\frac{1}{8 \lambda}\left(u_{1}-u_{2}\right) \\
& U_{i, j}^{n+1 / 2}=u_{i, j}^{n}-\lambda\left[F\left(u_{i+1, j}^{n}, u_{i, j}^{n},(n+1) \tau, x_{i+1 / 2}, y_{j}\right)\right. \\
& \left.-F\left(u_{i, j}^{n}, u_{i-1, j}^{n},(n+1) \tau, x_{i-1 / 2}, y_{j}\right)\right] \\
& U_{i, j}^{n+1}=U_{i, j}^{n+1 / 2}-\lambda\left[F\left(U_{i, j+1}^{n+1 / 2}, U_{i, j}^{n+1 / 2},(n+1) \tau, x_{i}, y_{j+1 / 2}\right)\right. \\
& \left.-F\left(U_{i, j}^{n+1 / 2}, U_{i, j-1}^{n+1 / 2},(n+1) \tau, x_{i}, y_{j-1 / 2}\right)\right] \\
& u_{i, j}^{n+1}=U_{i, j}^{n+1}\left[1+\tau\left(\alpha w_{i, j}^{n+1}-\beta\right)\left(1+\frac{\tau}{2}\left(\alpha w_{i, j}^{n+1}-\beta\right)\right)\right]
\end{aligned}
$$

end 
The flux numerical function $F\left(u_{1}, u_{2}, t, x, y\right)$, defined in (2.4d) involves the evaluation of the velocity function $\boldsymbol{v}$ at points $x_{i+1 / 2, j}$ and $x_{i, j+1 / 2}$. Due to the definition of $\boldsymbol{v}$ through a convolution of $w$ and a kernel function $\eta$, this necessities the numerical computation of $w^{n} * \eta$. Since the algorithm (2.4) is (at most) first order, we propose to use a standard quadrature formula on the same space mesh and compute

$$
\left(w^{n} * \eta\right)\left(x_{i}, y_{j}\right)=\sum_{k, \ell} h^{2} w_{k, \ell}^{n} \eta_{i-k, j-\ell}
$$

where $\eta_{i-k, j-\ell}=\eta\left(x_{i-k}, y_{j-\ell}\right)$. Notice that algorithm (2.4) needs values of $\boldsymbol{v}$ on the edges of each mesh element, such that we proceed as follows: whenever we are dealing with cells with the same $x$-coordinate, respectively $y$-coordinate, we average the corresponding component of $\boldsymbol{v}$ in the $x$-coordinate, respectively $y$-coordinate, thus obtaining

$$
\begin{gathered}
v^{n}\left(x_{i+1 / 2}, y_{j}\right)=\frac{1}{2}\left(v^{n}\left(x_{i}, y_{j}\right)+v^{n}\left(x_{i+1}, y_{j}\right)\right)=\frac{1}{2}\left[v\left(\left(w^{n} * \eta\right)\left(x_{i}, y_{j}\right)\right)+v\left(\left(w^{n} * \eta\right)\left(x_{i+1}, y_{j}\right)\right)\right] \\
v^{n}\left(x_{i}, y_{j+1 / 2}\right)=\frac{1}{2}\left(v^{n}\left(x_{i}, y_{j}\right)+v^{n}\left(x_{i}, y_{j+1}\right)\right)=\frac{1}{2}\left[v\left(\left(w^{n} * \eta\right)\left(x_{i}, y_{j}\right)\right)+v\left(\left(w^{n} * \eta\right)\left(x_{i}, y_{j+1}\right)\right)\right] .
\end{gathered}
$$

\section{Remark 2.2.}

- The values of $\eta_{i, j}$ can be computed in a preprocessing step and stored in a matrix. This reduces the computational cost for the evaluation of $\boldsymbol{v}$ to a matrix-vector multiplication at each point $x_{i, j}$.

- In principle, the above quadrature formula can also be used to evaluate $\left(w^{n} * \eta\right)\left(x_{i+1 / 2}, y_{j}\right)$ directly, using pointwise evaluations of $\eta$ at $\left(x_{i+1 / 2-k}, y_{j-l}\right)$. However, the double storage capacity for $\eta_{i+1 / 2, j}$ and $\eta_{i, j+1 / 2}$ will be needed for this strategy.

- If the kernel function $\eta$ admits an explicit form of the antiderivative, it is possible to compute the convolution $w^{n} * \eta$ exactly for piecewise constant $w^{n}$, since we have

$$
\left(w^{n} * \eta\right)\left(x_{i}, y_{j}\right):=\sum_{k, l} \int_{I_{k, l}} w_{k, l}^{n} \eta\left(x_{i}-x, y_{j}-y\right) \mathrm{d} x \mathrm{~d} y=\sum_{k, l} w_{k, l}^{n} \int_{I_{k, l}} \eta\left(x_{i}-x, y_{j}-y\right) \mathrm{d} x \mathrm{~d} y .
$$

Thus, storing the values of $\int_{I_{k, l}} \eta\left(x_{i}-x, y_{j}-y\right) \mathrm{d} x \mathrm{~d} y$ in a preprocessing step yields an exact evaluation of the convolution.

- Observe that the differentiation property of the convolution product still holds in the discrete case. Hence, if the function $\boldsymbol{v}$ satisfies (v) in the continuous case, this assumption holds also for its discrete approximation, obtained using the discrete convolution product, computed through formula (2.5), substituting $\eta$ by $\nabla \eta$.

Remark 2.3. All estimates of Section 3 as well as the convergence result of Section 4 can be shown analogously when $(2.4 \mathrm{e})$ and $(2.4 \mathrm{f})$ are replaced by

$$
\begin{array}{r}
U_{i, j}^{n+1}=u_{i, j}^{n}-\lambda\left[F\left(u_{i+1, j}^{n}, u_{i, j}^{n},(n+1) \tau, x_{i+1 / 2}, y_{j}\right)-F\left(u_{i, j}^{n}, u_{i-1, j}^{n},(n+1) \tau, x_{i-1 / 2}, y_{j}\right)\right. \\
\left.F\left(u_{i, j+1}^{n}, u_{i, j}^{n},(n+1) \tau, x_{i}, y_{j+1 / 2}\right)-F\left(u_{i, j}^{n}, u_{i, j-1}^{n},(n+1) \tau, x_{i}, y_{j-1 / 2}\right)\right] .
\end{array}
$$

Remark 2.4. The main reason for the choice of a non standard CFL condition as (2.1) is the space-time dependent velocity field $\boldsymbol{v}$. To prove the positivity of $u$ in Lemma 3.3 we observe that the space-time dependence of $\boldsymbol{v}$ introduces an additional constraint on $\lambda$. The choice of $1 / 4$ in the CFL condition and of $1 / 8$ in the definition of the Lax-Friedrichs flux are optimal in the sense that they provide the largest possible CFL condition that guarantees positivity of $u$. More details on this can be found in the proof of Lemma 3.3 in the next section. 


\section{BOUNDS ON $w$ AND $u$}

The proof of convergence of the above algorithm to the unique solution of (1.1) is based on an extension of Helly's theorem (see [10], Thm. 1.7.3). To apply this theorem, we have to prove the uniform boundedness of $u$ and $w$ in $\mathbf{L}^{1}$ as well as a uniform bound on the time-space total variation. The necessary estimates are collected in this section, starting with positivity estimates in Section 3.1 and bounds on the $\mathbf{L}^{1}$ and $\mathbf{L}^{\infty}$ norms in Section 3.2. Once these bounds are available, we are able to prove a bound on the total variation in space in Section 3.3. Finally, the Lipschitz continuity in time of $u$, proven in Section 3.4, guarantees enough regularity of the approximate solutions to pass to the convergence proof in Section 4.

In the sequel, we will make use of the following Lemma in several estimates.

Lemma 3.1. Let $a, b \in \mathbb{R}$, with $a, b>0$. The following inequality holds

$$
a \sum_{k=1}^{n} \mathrm{e}^{k a b} \leq \frac{1}{b} \mathrm{e}^{(n+1) a b}
$$

Proof. Recall that $a b+1 \leq \mathrm{e}^{a b}$. Hence,

$$
a \sum_{k=1}^{n} \mathrm{e}^{k a b}=a \frac{\mathrm{e}^{a b}\left(\mathrm{e}^{n a b}-1\right)}{\mathrm{e}^{a b}-1} \leq \frac{1}{b} \mathrm{e}^{(n+1) a b},
$$

concluding the proof.

Remark 3.2. In what follow we restrict ourselves to the case $\gamma>0$, in order to apply Lemma 3.1 for $b=\gamma$. However, a modification of the estimate in Lemma 3.1 to cover also the case $\gamma=0$ is possible, at the price of more complicated formulas in the estimates below.

\subsection{Positivity of $w$ and $u$}

Lemma 3.3. Let assumptions (f), (v) and (0) hold. Then the approximate solution constructed by Algorithm 2.1 is such that $w_{i, j}^{n} \geq 0$ and $u_{i, j}^{n} \geq 0$ for all $i, j$ and $n$.

Proof. Consider $w$ first, in particular, focus on the sequence $\left(W^{n, \ell}\right)$. Suppose $W_{i, j}^{n, \ell} \geq 0$ for all $i, j$ and define $S=S_{i, j}^{n}=\tau_{p}\left(\gamma-\delta u_{i, j}^{n}\right)$. By $(2.4 \mathrm{~b})$ we have

$$
W_{i, j}^{n, \ell+1}=\frac{1}{4}\left(W_{i+1, j}^{n, \ell}+W_{i-1, j}^{n, \ell}+W_{i, j+1}^{n, \ell}+W_{i, j-1}^{n, \ell}\right)\left(1+S+\frac{S^{2}}{2}\right) .
$$

The parabola $\left(1+S+S^{2} / 2\right)$ assumes only positive values and, by the inductive hypothesis, we deduce that $W_{i, j}^{n, \ell+1} \geq 0$. By induction, we can thus conclude that $w_{i, j}^{n} \geq 0$ for all $i, j$ and $n$.

Consider now $u$ and recall $v_{i+1 / 2, j}^{n+1}:=\boldsymbol{v}\left((n+1) \tau, x_{i+1 / 2}, y_{j}\right) \cdot \boldsymbol{n}_{i+1 / 2, j}$. By $(2.4 \mathrm{~d})$ and $(2.4 \mathrm{e})$ we have

$$
\begin{aligned}
U_{i, j}^{n+1 / 2}= & \frac{1}{8}\left(u_{i+1, j}^{n}+6 u_{i, j}^{n}+u_{i-1, j}^{n}\right)-\lambda f\left(u_{i, j}^{n}\right)\left(v_{i+1 / 2, j}^{n+1}-v_{i-1 / 2, j}^{n+1}\right) \\
& -\lambda\left[\frac{f\left(u_{i+1, j}^{n}\right)-f\left(u_{i, j}^{n}\right)}{2} v_{i+1 / 2, j}^{n+1}-\frac{f\left(u_{i-1, j}^{n}\right)-f\left(u_{i, j}^{n}\right)}{2} v_{i-1 / 2, j}^{n+1}\right] \\
= & u_{i+1, j}^{n}\left[\frac{1}{8}-\frac{\lambda}{2} \frac{f\left(u_{i+1, j}^{n}\right)-f\left(u_{i, j}^{n}\right)}{u_{i+1, j}^{n}-u_{i, j}^{n}} v_{i+1 / 2, j}^{n+1}\right]+u_{i-1, j}^{n}\left[\frac{1}{8}+\frac{\lambda}{2} \frac{f\left(u_{i-1, j}^{n}\right)-f\left(u_{i, j}^{n}\right)}{u_{i-1, j}^{n}-u_{i, j}^{n}} v_{i-1 / 2, j}^{n+1}\right] \\
& +u_{i, j}^{n}\left[\frac{3}{4}+\frac{\lambda}{2} v_{i+1 / 2, j}^{n+1}\left(\frac{f\left(u_{i+1, j}^{n}\right)-f\left(u_{i, j}^{n}\right)}{u_{i+1, j}^{n}-u_{i, j}^{n}}-2 \frac{f\left(u_{i, j}^{n}\right)}{u_{i, j}^{n}}\right)\right. \\
& \left.-\frac{\lambda}{2} v_{i-1 / 2, j}^{n+1}\left(\frac{f\left(u_{i-1, j}^{n}\right)-f\left(u_{i, j}^{n}\right)}{u_{i-1, j}^{n}-u_{i, j}^{n}}-2 \frac{f\left(u_{i, j}^{n}\right)}{u_{i, j}^{n}}\right)\right] .
\end{aligned}
$$


Observe that the CFL condition (2.1) yields

$$
\frac{1}{8} \pm \frac{\lambda}{2} \frac{f\left(u_{i+1, j}^{n}\right)-f\left(u_{i, j}^{n}\right)}{u_{i+1, j}^{n}-u_{i, j}^{n}} v_{i+1 / 2, j}^{n+1} \geq \frac{1}{8}-\frac{\lambda}{2}\left\|\partial_{u} f\right\|_{\mathbf{L}^{\infty}}\|v\|_{\mathbf{L}^{\infty}}>0,
$$

and for the remaining term

$$
\begin{aligned}
& \frac{3}{4}+\frac{\lambda}{2} v_{i+1 / 2, j}^{n+1}\left[\frac{f\left(u_{i+1, j}^{n}\right)-f\left(u_{i, j}^{n}\right)}{u_{i+1, j}^{n}-u_{i, j}^{n}}-2 \frac{f\left(u_{i, j}^{n}\right)}{u_{i, j}^{n}}\right]-\frac{\lambda}{2} v_{i-1 / 2, j}^{n+1}\left[\frac{f\left(u_{i-1, j}^{n}\right)-f\left(u_{i, j}^{n}\right)}{u_{i-1, j}^{n}-u_{i, j}^{n}}-2 \frac{f\left(u_{i, j}^{n}\right)}{u_{i, j}^{n}}\right] \\
\geq & \frac{3}{4}-3 \lambda\|v\|_{\mathbf{L}^{\infty}}\left\|\partial_{u} f\right\|_{\mathbf{L}^{\infty}}>0 .
\end{aligned}
$$

Hence, using also the inductive hypothesis, we have that $U_{i, j}^{n+1 / 2} \geq 0$ for all $i$ and $j$.

Using (2.4f), we can repeat the same steps as above considering $U^{n+1 / 2}$ instead of $u^{n}$ to conclude that $U_{i, j}^{n+1} \geq 0$ for all $i$ and $j$.

Finally, defining $R=R_{i, j}^{n+1}=\tau\left(\alpha w_{i, j}^{n+1}-\beta\right)$ and using (2.4g), we obtain

$$
u_{i, j}^{n+1}=U_{i, j}^{n+1}\left(1+R+\frac{R^{2}}{2}\right) .
$$

Analogously to $w$, we can conclude that $u_{i, j}^{n+1} \geq 0$ for all $i, j$ and $n$.

\section{2. $\mathrm{L}^{\infty}$ and $\mathrm{L}^{1}$ bounds on $w$ and $u$}

Lemma 3.4. Let assumptions (f), (v) and (0) hold. Then for all $n$ the approximate solution $\left(u^{n}, w^{n}\right)$ constructed by Algorithm 2.1 satisfies

$$
\begin{aligned}
\left\|w^{n}\right\|_{\mathbf{L}^{\infty}} & \leq \mathrm{e}^{n \tau \gamma}\left\|w^{o}\right\|_{\mathbf{L}^{\infty}} \\
\left\|u^{n}\right\|_{\mathbf{L}^{\infty}} & \leq\left\|u^{o}\right\|_{\mathbf{L}^{\infty}} \exp \left(\left(2 \mathcal{K}_{1}+\mathcal{K}_{2}\right) \frac{1}{\gamma} \mathrm{e}^{(n+1) \tau \gamma}\right),
\end{aligned}
$$

where $\mathcal{K}_{1}, \mathcal{K}_{2}$ are constants depending on $\alpha, K,\left\|w^{o}\right\|_{\mathbf{L}^{\infty}},\left\|\partial_{u} f\right\|_{\mathbf{L}^{\infty}}$.

Proof. Consider $w$ first. By Lemma 3.3, $u_{i, j}^{n} \geq 0$ for all $i, j$ and $n$. By $(2.4 \mathrm{~b})$ we have for $0 \leq l<m$

$$
\begin{aligned}
W_{i, j}^{n, l+1} & \leq \mathrm{e}^{\tau_{p}\left(\gamma-\delta u_{i, j}^{n}\right)} \frac{1}{4}\left(W_{i+1, j}^{n, l}+W_{i-1, j}^{n, l}+W_{i, j+1}^{n, l}+W_{i, j-1}^{n, l}\right) \\
& \leq \mathrm{e}^{\tau_{p} \gamma}\left\|W^{n, l}\right\|_{\mathbf{L}^{\infty}} .
\end{aligned}
$$

By induction over $l$ in the sequence $W^{n, l}$ we obtain therefore

$$
\left\|w^{n+1}\right\|_{\mathbf{L}^{\infty}}=\left\|W^{n, m}\right\|_{\mathbf{L}^{\infty}} \leq \mathrm{e}^{m \tau_{p} \gamma}\left\|w^{n}\right\|_{\mathbf{L}^{\infty}}=\mathrm{e}^{\tau \gamma}\left\|w^{n}\right\|_{\mathbf{L}^{\infty}}
$$

Finally, induction over $n$ yields

$$
\left\|w^{n}\right\|_{\mathbf{L}^{\infty}} \leq \mathrm{e}^{n \tau \gamma}\left\|w^{o}\right\|_{\mathbf{L}^{\infty}} .
$$

Pass now to $u$ and recall that by (v), we have

$$
\left\|\nabla v^{n}\right\|_{\mathbf{L}^{\infty}} \leq K\left\|w^{n}\right\|_{\mathbf{L}^{\infty}} .
$$


As in Lemma 3.3, by (2.4d) and (2.4e), simple computations lead to

$$
\begin{aligned}
\left|U_{i, j}^{n+1 / 2}\right|= & U_{i, j}^{n+1 / 2} \\
= & u_{i+1, j}^{n}\left[\frac{1}{8}-\frac{\lambda}{2} \frac{f\left(u_{i+1, j}^{n}\right)-f\left(u_{i}^{n}\right)}{u_{i+1, j}^{n}-u_{i, j}^{n}} v_{i+1 / 2, j}^{n+1}\right]+u_{i-1, j}^{n}\left[\frac{1}{8}+\frac{\lambda}{2} \frac{f\left(u_{i-1, j}^{n}\right)-f\left(u_{i}^{n}\right)}{u_{i-1, j}^{n}-u_{i, j}^{n}} v_{i-1 / 2, j}^{n+1}\right] \\
& +u_{i, j}^{n}\left[\frac{3}{4}+\frac{\lambda}{2} \frac{f\left(u_{i+1, j}^{n}\right)-f\left(u_{i, j}^{n}\right)}{u_{i+1, j}^{n}-u_{i, j}^{n}} v_{i+1 / 2, j}^{n+1}-\frac{\lambda}{2} \frac{f\left(u_{i-1, j}^{n}\right)-f\left(u_{i, j}^{n}\right)}{u_{i-1, j}^{n}-u_{i, j}^{n}} v_{i-1 / 2, j}^{n+1}\right. \\
& \left.-\lambda \frac{f\left(u_{i, j}^{n}\right)}{u_{i, j}^{n}}\left(v_{i+1 / 2, j}^{n+1}-v_{i-1 / 2, j}^{n+1}\right)\right] \\
\leq & \left\|u^{n}\right\|_{\mathbf{L}^{\infty}}\left[\frac{1}{8}-\frac{\lambda}{2} \frac{f\left(u_{i+1, j}^{n}\right)-f\left(u_{i, j}^{n}\right)}{u_{i+1, j}^{n}-u_{i, j}^{n}} v_{i+1 / 2, j}^{n+1}+\frac{1}{8}+\frac{\lambda}{2} \frac{f\left(u_{i-1, j}^{n}\right)-f\left(u_{i, j}^{n}\right)}{u_{i-1, j}^{n}-u_{i, j}^{n}} v_{i-1 / 2, j}^{n+1}\right. \\
& +\frac{3}{4}+\frac{\lambda}{2} \frac{f\left(u_{i+1, j}^{n}\right)-f\left(u_{i, j}^{n}\right)}{u_{i+1, j}^{n}-u_{i, j}^{n}} v_{i+1 / 2, j}^{n+1}-\frac{\lambda}{2} \frac{f\left(u_{i-1, j}^{n}\right)-f\left(u_{i, j}^{n}\right)}{u_{i-1, j}^{n}-u_{i, j}^{n}} v_{i-1 / 2, j}^{n+1} \\
& \left.-\lambda \frac{f\left(u_{i, j}^{n}\right)}{u_{i, j}^{n}}\left(v_{i+1 / 2, j}^{n+1}-v_{i-1 / 2, j}^{n+1}\right)\right] \\
\leq & \left\|u^{n}\right\|_{\mathbf{L}_{\infty}^{\infty}}\left(1+\tau\left\|\partial_{u} f\right\|_{\mathbf{L}^{\infty}}\left\|\partial_{x} v^{n+1}\right\|_{\mathbf{L}^{\infty}}\right) \\
\leq & \left\|u^{n}\right\|_{\mathbf{L}^{\infty}} \exp \left(\tau K\left\|\partial_{u} f\right\|_{\mathbf{L}^{\infty}}\left\|w^{n+1}\right\|_{\mathbf{L}^{\infty}}\right) .
\end{aligned}
$$

The estimate on $U^{n+1}$ can be obtained analogously using (2.4f) to get

$$
\left\|U^{n+1}\right\|_{\mathbf{L}^{\infty}} \leq\left\|U^{n+1 / 2}\right\|_{\mathbf{L}^{\infty}} \exp \left(\tau K\left\|\partial_{u} f\right\|_{\mathbf{L}^{\infty}}\left\|w^{n+1}\right\|_{\mathbf{L}^{\infty}}\right) .
$$

Concerning the source term, integrated by $(2.4 \mathrm{~g})$, one can easily see that

$$
\left|u_{i, j}^{n+1}\right| \leq U_{i, j}^{n+1} \exp \left[\tau\left(\alpha w_{i, j}^{n+1}-\beta\right)\right] \leq\left\|U^{n+1}\right\|_{\mathbf{L}^{\infty}} \exp \left(\tau \alpha\left\|w^{n+1}\right\|_{\mathbf{L}^{\infty}}\right) .
$$

Collecting the above estimates and using (3.1), we conclude that

$$
\left\|u^{n+1}\right\|_{\mathbf{L}^{\infty}} \leq\left\|u^{n}\right\|_{\mathbf{L}^{\infty}} \exp \left[\tau \mathrm{e}^{(n+1) \tau \gamma}\left\|w^{o}\right\|_{\mathbf{L}^{\infty}}\left(2 K\left\|\partial_{u} f\right\|_{\mathbf{L}^{\infty}}+\alpha\right)\right] .
$$

Iterating over $n$ and applying Lemma 3.1 yields

$$
\begin{aligned}
\left\|u^{n}\right\|_{\mathbf{L}^{\infty}} & \leq\left\|u^{o}\right\|_{\mathbf{L}^{\infty}} \exp \left[\tau\left\|w^{o}\right\|_{\mathbf{L}^{\infty}}\left(2 K\left\|\partial_{u} f\right\|_{\mathbf{L}^{\infty}}+\alpha\right) \sum_{k=1}^{n} \mathrm{e}^{k \tau \gamma}\right] \\
& \leq\left\|u^{o}\right\|_{\mathbf{L}^{\infty}} \exp \left[\mathrm{e}^{(n+1) \tau \gamma} \frac{1}{\gamma}\left\|w^{o}\right\|_{\mathbf{L}^{\infty}}\left(2 K\left\|\partial_{u} f\right\|_{\mathbf{L}^{\infty}}+\alpha\right)\right] .
\end{aligned}
$$

Denoting

$$
\mathcal{K}_{1}=K\left\|w^{o}\right\|_{\mathbf{L}^{\infty}}\left\|\partial_{u} f\right\|_{\mathbf{L}^{\infty}} \quad \mathcal{K}_{2}=\alpha\left\|w^{o}\right\|_{\mathbf{L}^{\infty}}
$$

completes the proof.

Positivity and uniform boundedness of the approximate solution allow now to prove the $\mathbf{L}^{1}$ bounds necessary for the application of ([10], Thm. 1.7.3) in the convergence proof later on. 
Lemma 3.5. Let assumptions (f), (v) and (0) hold. Then for all $n$ the approximate solution $\left(u^{n}, w^{n}\right)$ constructed by Algorithm 2.1 satisfies

$$
\begin{aligned}
\left\|w^{n}\right\|_{\mathbf{L}^{1}} & \leq \mathrm{e}^{n \tau \gamma}\left\|w^{o}\right\|_{\mathbf{L}^{1}} \\
\left\|u^{n}\right\|_{\mathbf{L}^{1}} & \leq\left\|u^{o}\right\|_{\mathbf{L}^{1}} \exp \left(\mathcal{K}_{2} \frac{1}{\gamma} \mathrm{e}^{(n+1) \tau \gamma}\right),
\end{aligned}
$$

where $\mathcal{K}_{2}$ is the constant defined in Lemma 3.4 , depending on $\alpha,\left\|w^{o}\right\|_{\mathbf{L}^{\infty}}$.

Proof. Consider $w$ first. By Lemma 3.3, $u_{i, j}^{n} \geq 0$ and $w_{i, j}^{n} \geq 0$ for all $i, j$ and $n$. Let $W^{n, 0}=w^{n}$ and $0 \leq l<m$. By (2.4b),

$$
\left\|W^{n, l+1}\right\|_{\mathbf{L}^{1}}=\sum_{i \in \mathbb{Z}} \sum_{j \in \mathbb{Z}} h^{2} W_{i, j}^{n, l+1} \leq \mathrm{e}^{\tau_{p}\left(\gamma-\delta u_{i, j}^{n}\right)} \sum_{i \in \mathbb{Z}} \sum_{j \in \mathbb{Z}} h^{2} W_{i, j}^{n, l} \leq \mathrm{e}^{\tau_{p} \gamma}\left\|W^{n, l}\right\|_{\mathbf{L}^{1}} .
$$

Induction over $l$ yields

$$
\left\|w^{n+1}\right\|_{\mathbf{L}^{1}}=\left\|W^{n, m}\right\|_{\mathbf{L}^{1}} \leq \mathrm{e}^{m \tau_{p} \gamma}\left\|w^{n}\right\|_{\mathbf{L}^{1}} \leq \mathrm{e}^{\tau \gamma}\left\|w^{n}\right\|_{\mathbf{L}^{1}} .
$$

Induction over $n$ now yields

$$
\left\|w^{n}\right\|_{\mathbf{L}^{1}} \leq \mathrm{e}^{n \tau \gamma}\left\|w^{o}\right\|_{\mathbf{L}^{1}}
$$

Pass now to $u$. By the conservation property of the Lax-Friedrichs scheme (2.4d)-(2.4f) we have

$$
\left\|U^{n+1}\right\|_{\mathbf{L}^{1}}=\left\|U^{n+1 / 2}\right\|_{\mathbf{L}^{1}}=\left\|u^{n}\right\|_{\mathbf{L}^{1}} .
$$

To include the source term in the $\mathbf{L}^{1}$-estimate, we consider (2.4g) and obtain

$$
\begin{aligned}
& \left\|u^{n+1}\right\|_{\mathbf{L}^{1}}=\sum_{i \in \mathbb{Z}} \sum_{j \in \mathbb{Z}} h^{2} u_{i, j}^{n+1} \leq \sum_{i \in \mathbb{Z}} \sum_{j \in \mathbb{Z}} h^{2} \mathrm{e}^{\tau\left(\alpha w_{i, j}^{n+1}-\beta\right)} U_{i, j}^{n+1} \\
& \leq \mathrm{e}^{\tau \alpha \| w^{n+1}} \|_{\mathbf{L} \infty} \sum_{i \in \mathbb{Z}} \sum_{j \in \mathbb{Z}} h^{2} U_{i, j}^{n+1} \\
& =\mathrm{e}^{\tau \alpha \| w^{n+1}}\left\|_{\mathbf{L}^{\infty}}\right\| u^{n} \|_{\mathbf{L}^{1}} \text {. }
\end{aligned}
$$

Using (3.1) and (3.3), this yields

$$
\begin{aligned}
\left\|u^{n+1}\right\|_{\mathbf{L}^{1}} & \leq\left\|u^{n}\right\|_{\mathbf{L}^{1}} \exp \left(\tau \alpha\left\|w^{o}\right\|_{\mathbf{L}^{\infty}} \mathrm{e}^{(n+1) \tau \gamma}\right) \\
& \leq\left\|u^{o}\right\|_{\mathbf{L}^{1}} \exp \left(\tau \mathcal{K}_{2} \sum_{k=1}^{n+1} \mathrm{e}^{k \tau \gamma}\right) \\
& \leq\left\|u^{o}\right\|_{\mathbf{L}^{1}} \exp \left(\mathcal{K}_{2} \frac{1}{\gamma} \mathrm{e}^{(n+2) \tau \gamma}\right),
\end{aligned}
$$

where we applied Lemma 3.1. This completes the proof.

\subsection{TV estimate}

Lemma 3.6. Let assumptions (f), (v) and (0) hold and fix $0<T<\infty$. Then, for all $n$ such that $n \tau<T$, the approximate solution $\left(u^{n}, w^{n}\right)$ constructed by Algorithm 2.1 satisfies

$$
\operatorname{TV}\left(u^{n}\right)+\operatorname{TV}\left(w^{n}\right) \leq \mathrm{e}^{n \tau \mathcal{K}_{3}}\left(\operatorname{TV}\left(u^{o}\right)+\operatorname{TV}\left(w^{o}\right)+\frac{\mathcal{K}_{4}}{\mathcal{K}_{3}} \mathrm{e}^{\tau \mathcal{K}_{5}}\right)
$$

where the functions $\mathcal{K}_{i}, i=3, \ldots, 5$ depend on $T$, various norms of $u^{n}, w^{n}$ and $\partial_{u} f$ as well as on all constants $\alpha, \beta, \gamma, \delta$ and $K$ and the function $C$ defined in $(\mathbf{v})$. 
Proof. Consider $w$ first. In particular, focus on $W^{n, l}$ defined in (2.4b). To obtain a bound on the total variation of $w^{n+1}$, we have to estimate

$$
\operatorname{TV}\left(w^{n+1}\right)=\sum_{i \in \mathbb{Z}} \sum_{j \in \mathbb{Z}} h\left[\left|w_{i+1, j}^{n+1}-w_{i, j}^{n+1}\right|+\left|w_{i, j+1}^{n+1}-w_{i, j}^{n+1}\right|\right] .
$$

Similarly as before, we define $S_{i, j}^{n}=\gamma-\delta u_{i, j}^{n}$ for the sake of simplicity. To obtain a bound for (3.6), we consider

$$
\begin{aligned}
& \sum_{i, j} h\left|W_{i+1, j}^{n, l+1}-W_{i, j}^{n, l+1}\right| \\
\leq & \frac{1}{4} \mathrm{e}^{\tau_{p} \gamma} \sum_{i, j} h\left(\left|W_{i+2, j}^{n, l}-W_{i+1, j}^{n, l}\right|+\left|W_{i, j}^{n, l}-W_{i-1, j}^{n, l}\right|+\left|W_{i+1, j+1}^{n, l}-W_{i, j+1}^{n, l}\right|+\left|W_{i+1, j-1}^{n, l}-W_{i, j-1}^{n, l}\right|\right) \\
& +\sum_{i, j} h\left|W_{i, j}^{n, l}\right| \cdot \tau_{p}\left|S_{i+1, j}^{n}\left(1+\frac{\tau_{p}}{2} S_{i+1, j}^{n}\right)-S_{i, j}^{n}\left(1+\frac{\tau_{p}}{2} S_{i, j}^{n}\right)\right| \\
\leq & \mathrm{e}^{\tau_{p} \gamma} \sum_{i, j} h\left|W_{i, j}^{n, l}-W_{i-1, j}^{n, l}\right|+\left\|W^{n, l}\right\|_{\mathbf{L}^{\infty}} \sum_{i, j} h \tau_{p}\left|S_{i+1, j}^{n}-S_{i, j}^{n}\right| \cdot\left|1+\tau_{p}\left(\gamma-\frac{\delta}{2}\left(u_{i+1, j}^{n}+u_{i, j}^{n}\right)\right)\right| \\
\leq & \mathrm{e}^{\tau_{p} \gamma} \sum_{i, j} h\left|W_{i, j}^{n, l}-W_{i-1, j}^{n, l}\right|+\tau_{p}\left(1+\tau_{p}\left(\gamma+\delta\left\|u^{n}\right\|_{\mathbf{L}^{\infty}}\right)\right) \delta\left\|W^{n, l}\right\|_{\mathbf{L}_{\infty}^{\infty}} \cdot \sum_{i, j} h\left|u_{i+1, j}^{n}-u_{i, j}^{n}\right| \\
\leq & \mathrm{e}^{\tau_{p} \gamma}\left(\sum_{i, j} h\left|W_{i, j}^{n, l}-W_{i-1, j}^{n, l}\right|+\tau_{p} \mathrm{e}^{\delta\left\|u^{n}\right\|_{\mathbf{L} \infty} \tau_{p}} \delta\left\|W^{n, l}\right\|_{\mathbf{L}^{\infty}} \sum_{i, j} h\left|u_{i+1, j}^{n}-u_{i, j}^{n}\right|\right) .
\end{aligned}
$$

An analogous estimate can be derived for $\sum_{i, j} h\left|W_{i, j+1}^{n, l+1}-W_{i, j}^{n, l+1}\right|$. Induction over $l$ yields now

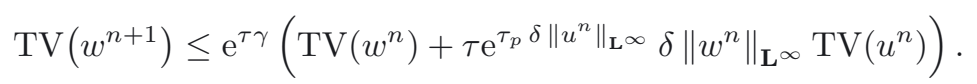

Pass now to $u$. We need to estimate the following quantity:

$$
\operatorname{TV}\left(u^{n+1}\right)=h \sum_{i \in \mathbb{Z}} \sum_{j \in \mathbb{Z}}\left[\left|u_{i+1, j}^{n+1}-u_{i, j}^{n+1}\right|+\left|u_{i, j+1}^{n+1}-u_{i, j}^{n+1}\right|\right] .
$$

Denoting $R_{i, j}^{n+1}=\alpha w_{i, j}^{n+1}-\beta$ for the sake of simplicity, we obtain

$$
\begin{aligned}
\operatorname{TV}\left(u^{n+1}\right)= & \sum_{i, j} h\left[\left|u_{i+1, j}^{n+1}-u_{i, j}^{n+1}\right|+\left|u_{i, j+1}^{n+1}-u_{i, j}^{n+1}\right|\right] \\
\leq & \mathrm{e}^{\tau \alpha\left\|w^{n+1}\right\|_{\mathbf{L}}} \sum_{i, j} h\left[\left|U_{i+1, j}^{n+1}-U_{i, j}^{n+1}\right|+\left|U_{i, j+1}^{n+1}-U_{i, j}^{n+1}\right|\right] \\
& +\left\|U^{n+1}\right\|_{\mathbf{L}^{\infty}} \tau \sum_{i, j} h\left|R_{i+1, j}^{n+1}\left(1+\frac{\tau}{2} R_{i+1, j}^{n+1}\right)-R_{i, j}^{n+1}\left(1+\frac{\tau}{2} R_{i, j}^{n+1}\right)\right| \\
& +\left\|U^{n+1}\right\|_{\mathbf{L}^{\infty}} \tau \sum_{i, j} h\left|R_{i, j+1}^{n+1}\left(1+\frac{\tau}{2} R_{i, j+1}^{n+1}\right)-R_{i, j}^{n+1}\left(1+\frac{\tau}{2} R_{i, j}^{n+1}\right)\right| \\
\leq & \mathrm{e}^{\tau \alpha\left\|w^{n+1}\right\|_{\mathbf{L} \infty}}\left(\operatorname{TV}\left(U^{n+1}\right)+\tau \alpha \mathrm{e}^{\tau \beta}\left\|U^{n+1}\right\|_{\mathbf{L}^{\infty}} \operatorname{TV}\left(w^{n+1}\right)\right) .
\end{aligned}
$$

To approximate $\mathrm{TV}\left(U^{n+1}\right)$, we have to estimate

$$
\sum_{i, j} h\left(\left|U_{i+1, j}^{n+1}-U_{i, j}^{n+1}\right|+\left|U_{i, j+1}^{n+1}-U_{i, j}^{n+1}\right|\right) .
$$


It is a well-known fact that the standard Lax-Friedrichs scheme is TVD and thus TV $\left(U^{n+1}\right) \leq \mathrm{TV}\left(U^{n+1 / 2}\right) \leq$ TV $\left(u^{n}\right)$. The situation here however is different, since the flux does not only depend on $u$, but also on $t$ and $x$ through the component $v(w)$. The conservation law itself does therefore not satisfy the TVD-property (see [8]) and we cannot expect the numerical scheme to be TVD. To estimate the increase in total variation due to the space-time dependent velocity field, we consider the term $\sum_{i, j} h\left|U_{i, j+1}^{n+1}-U_{i, j}^{n+1}\right|$. By (2.4f), we have

$$
\begin{aligned}
U_{i, j+1}^{n+1}-U_{i, j}^{n+1} & =U_{i, j+1}^{n+1 / 2}-U_{i, j}^{n+1 / 2} \\
-\lambda\left[F\left(U_{i, j+2}^{n+1 / 2}, U_{i, j+1}^{n+1 / 2},(n+1) \tau, x_{i, j+3 / 2}\right)-F\left(U_{i, j+1}^{n+1 / 2}, U_{i, j}^{n+1 / 2},(n+1) \tau, x_{i, j+1 / 2}\right)\right. & \left.\quad-F\left(U_{i, j+1}^{n+1 / 2}, U_{i, j}^{n+1 / 2},(n+1) \tau, x_{i, j+1 / 2}\right)+F\left(U_{i, j}^{n+1 / 2}, U_{i, j-1}^{n+1 / 2},(n+1) \tau, x_{i, j-1 / 2}\right)\right] .
\end{aligned}
$$

Add and subtract $\lambda F\left(U_{i, j+1}^{n+1 / 2}, U_{i, j}^{n+1 / 2},(n+1) \tau, x_{i, j+3 / 2}\right)+\lambda F\left(U_{i, j}^{n+1 / 2}, U_{i, j-1}^{n+1 / 2},(n+1) \tau, x_{i, j+1 / 2}\right)$, then rearrange to obtain:

$$
U_{i, j+1}^{n+1}-U_{i, j}^{n+1}=\mathcal{A}_{i, j}^{n}-\mathcal{B}_{i, j}^{n}
$$

where

$$
\begin{aligned}
\mathcal{A}_{i, j}^{n}= & U_{i, j+1}^{n+1 / 2}-U_{i, j}^{n+1 / 2} \\
- & \lambda\left[F\left(U_{i, j+2}^{n+1 / 2}, U_{i, j+1}^{n+1 / 2},(n+1) \tau, x_{i, j+3 / 2}\right)-F\left(U_{i, j+1}^{n+1 / 2}, U_{i, j}^{n+1 / 2},(n+1) \tau, x_{i, j+1 / 2}\right)\right. \\
+ & \left.F\left(U_{i, j}^{n+1 / 2}, U_{i, j-1}^{n+1 / 2},(n+1) \tau, x_{i, j+1 / 2}\right)-F\left(U_{i, j+1}^{n+1 / 2}, U_{i, j}^{n+1 / 2},(n+1) \tau, x_{i, j+3 / 2}\right)\right], \\
\mathcal{B}_{i, j}^{n}=\lambda & \lambda F\left(U_{i, j+1}^{n+1 / 2}, U_{i, j}^{n+1 / 2},(n+1) \tau, x_{i, j+3 / 2}\right)-F\left(U_{i, j+1}^{n+1 / 2}, U_{i, j}^{n+1 / 2},(n+1) \tau, x_{i, j+1 / 2}\right) \\
& \left.+F\left(U_{i, j}^{n+1 / 2}, U_{i, j-1}^{n+1 / 2},(n+1) \tau, x_{i, j-1 / 2}\right)-F\left(U_{i, j}^{n+1 / 2}, U_{i, j-1}^{n+1 / 2},(n+1) \tau, x_{i, j+1 / 2}\right)\right] .
\end{aligned}
$$

From now on we omit the superscripts, $n+1 / 2$ or $n+1$, to enhance readability. Consider first the term $\mathcal{A}_{i, j}^{n}$ and use $(2.4 \mathrm{~d})$ to obtain

$$
\begin{aligned}
\mathcal{A}_{i, j}^{n}= & U_{i, j+1}-U_{i, j}-\lambda\left[\frac{f\left(U_{i, j+2}\right)+f\left(U_{i, j+1}\right)-f\left(U_{i, j+1}\right)-f\left(U_{i, j}\right)}{2} v_{i, j+3 / 2}\right. \\
& \left.-\frac{f\left(U_{i, j+1}\right)+f\left(U_{i, j}\right)-f\left(U_{i, j}\right)-f\left(U_{i, j-1}\right)}{2} v_{i, j+1 / 2}\right] \\
& +\frac{1}{8}\left(\left(U_{i, j+2}-U_{i, j+1}\right)-2\left(U_{i, j+1}-U_{i, j}\right)+\left(U_{i, j}-U_{i, j-1}\right)\right) \\
= & \frac{3}{4}\left(U_{i, j+1}-U_{i, j}\right)+\left(U_{i, j+2}-U_{i, j+1}\right)\left(\frac{1}{8}-\frac{\lambda}{2} \frac{f\left(U_{i, j+2}\right)-f\left(U_{i, j+1}\right)}{U_{i, j+2}-U_{i, j+1}} v_{i, j+3 / 2}\right) \\
& +\left(U_{i, j}-U_{i, j-1}\right)\left(\frac{1}{8}+\frac{\lambda}{2} \frac{f\left(U_{i, j}\right)-f\left(U_{i, j-1}\right)}{U_{i, j}-U_{i, j-1}} v_{i, j+1 / 2}\right) \\
& -\frac{\lambda}{2}\left[f\left(U_{i, j+1}\right)-f\left(U_{i, j}\right)\right]\left(v_{i, j+3 / 2}-v_{i, j+1 / 2}\right) .
\end{aligned}
$$


Observe that both coefficients of $U_{i, j+2}-U_{i, j+1}$ and $U_{i, j}-U_{i, j-1}$ are positive. Then, summing the modulus of $\mathcal{A}_{i, j}^{n}$ over $i, j \in \mathbb{Z}$, using also (v), (3.1) and (3.3), yields:

$$
\begin{aligned}
\sum_{i, j} h\left|\mathcal{A}_{i, j}^{n}\right| \leq & \sum_{i, j} h\left|U_{i, j+1}-U_{i, j}\right|\left(1+\frac{\lambda}{2} \frac{f\left(U_{i, j+1}\right)-f\left(U_{i, j}\right)}{U_{i, j+1}-U_{i, j}}\left(v_{i, j+1 / 2}-v_{i, j+3 / 2}\right)\right) \\
& +\sum_{i, j} h \frac{\lambda}{2}\left|U_{i, j+1}-U_{i, j}\right|\left|\frac{f\left(U_{i, j+1}\right)-f\left(U_{i, j}\right)}{U_{i, j+1}-U_{i, j}}\right|\left|v_{i, j+3 / 2}-v_{i, j+1 / 2}\right| \\
\leq & \sum_{i, j} h\left|U_{i, j+1}^{n+1 / 2}-U_{i, j}^{n+1 / 2}\right|\left(1+\tau\left\|\partial_{u} f\right\|_{\mathbf{L}^{\infty}}\left\|\partial_{y} v^{n+1}\right\|_{\mathbf{L}^{\infty}}\right) \\
\leq & \sum_{i, j} h\left|U_{i, j+1}^{n+1 / 2}-U_{i, j}^{n+1 / 2}\right|\left(1+\tau K\left\|\partial_{u} f\right\|_{\mathbf{L}^{\infty}}\left\|w^{n+1}\right\|_{\mathbf{L}^{\infty}}\right) .
\end{aligned}
$$

Pass now to $\mathcal{B}_{i, j}^{n}$. We continue omitting the superscripts.

$$
\begin{aligned}
\mathcal{B}_{i, j}^{n}= & \lambda\left[\frac{f\left(U_{i, j}\right)+f\left(U_{i, j+1}\right)}{2} v_{i, j+3 / 2}^{n+1}-\frac{f\left(U_{i, j}\right)+f\left(U_{i, j+1}\right)}{2} v_{i, j+1 / 2}^{n+1}\right. \\
& \left.+\frac{f\left(U_{i, j-1}\right)+f\left(U_{i, j}\right)}{2} v_{i, j-1 / 2}^{n+1}-\frac{f\left(U_{i, j-1}\right)+f\left(U_{i, j}\right)}{2} v_{i, j+1 / 2}^{n+1}\right] \\
= & \frac{\lambda}{2}\left[f\left(U_{i, j}\right)\left(v_{i, j+3 / 2}^{n+1}-2 v_{i, j+1 / 2}^{n+1}+v_{i, j-1 / 2}^{n+1}\right)\right. \\
& \left.+\left(f\left(U_{i, j+1}\right)-f\left(U_{i, j-1}\right)\right)\left(v_{i, j+3 / 2}^{n+1}-v_{i, j+1 / 2}^{n+1}\right)+f\left(U_{i, j-1}\right)\left(v_{i, j+3 / 2}^{n+1}-2 v_{i, j+1 / 2}^{n+1}+v_{i, j-1 / 2}^{n+1}\right)\right] .
\end{aligned}
$$

Since $v=v_{2}$ is a smooth function and recalling that $v_{i, j+1 / 2}^{n+1}=\boldsymbol{v}\left((n+1) \tau, x_{i}, y_{j+1 / 2}\right) \cdot \boldsymbol{n}_{i, j+1 / 2}$ is the $y$-component of $\boldsymbol{v}$ at time $(n+1) \tau$ and point $x_{i, j+1 / 2}$, we obtain

$$
v_{i, j+3 / 2}^{n+1}-2 v_{i, j+1 / 2}^{n+1}+v_{i, j-1 / 2}^{n+1} \leq h^{2}\left\|\partial_{y y} v^{n+1}\right\|_{\mathbf{L}^{\infty}} .
$$

Then, using also (v),

$$
\frac{\lambda}{2} f\left(U_{i, j}^{n+1 / 2}\right)\left(v_{i, j+3 / 2}^{n+1}-2 v_{j+1 / 2}^{n+1}+v_{j-1 / 2}^{n+1}\right) \leq \frac{\lambda}{2} h^{2}\left\|\partial_{u} f\right\|_{\mathbf{L}^{\infty}}\left|U_{i, j}^{n+1 / 2}\right| C\left(\left\|w^{n+1}\right\|_{\mathbf{L}^{\infty}}\right),
$$

and similarly for the term with $f\left(U_{i, j-1}\right)$, while the remaining term can be easily estimated as follows

$$
\frac{\lambda}{2}\left(f\left(U_{i, j+1}^{n+1 / 2}\right)-f\left(U_{i, j-1}^{n+1 / 2}\right)\right)\left(v_{i, j+3 / 2}^{n+1}-v_{i, j+1 / 2}^{n+1}\right) \leq \frac{\lambda}{2}\left\|\partial_{u} f\right\|_{\mathbf{L}^{\infty}}\left|U_{i, j+1}^{n+1 / 2}-U_{i, j-1}^{n+1 / 2}\right| h\left\|\partial_{y} v^{n+1}\right\|_{\mathbf{L}^{\infty}} .
$$

Hence, multiplying by $h$ and summing over $i$ and $j,(3.12)$ and (3.13) yield

$$
\sum_{i, j} h\left|\mathcal{B}_{i, j}^{n}\right| \leq \tau\left\|\partial_{u} f\right\|_{\mathbf{L}^{\infty}}\left(K\left\|w^{n+1}\right\|_{\mathbf{L}^{\infty}} \sum_{i, j} h\left|U_{i, j+1}^{n+1 / 2}-U_{i, j}^{n+1 / 2}\right|+C\left(\left\|w^{n+1}\right\|_{\mathbf{L}^{\infty}}\right)\left\|u^{n}\right\|_{\mathbf{L}^{1}}\right) .
$$

By (3.11) and (3.14) we have

$$
\begin{aligned}
\sum_{i, j} h\left|U_{i, j+1}^{n+1}-U_{i, j}^{n+1}\right| \leq & \sum_{i, j} h\left|U_{i, j+1}^{n+1 / 2}-U_{i, j}^{n+1 / 2}\right|\left(1+2 \tau K\left\|\partial_{u} f\right\|_{\mathbf{L}^{\infty}}\left\|w^{n+1}\right\|_{\mathbf{L}^{\infty}}\right) \\
& +\tau\left\|\partial_{u} f\right\|_{\mathbf{L}^{\infty}} C\left(\left\|w^{n+1}\right\|_{\mathbf{L}^{\infty}}\right)\left\|u^{n}\right\|_{\mathbf{L}^{1}} .
\end{aligned}
$$


In a similar way we obtain

$$
\begin{aligned}
\sum_{i, j} h\left|U_{i+1, j}^{n+1}-U_{i, j}^{n+1}\right| \leq & \sum_{i, j} h\left|U_{i+1, j}^{n+1 / 2}-U_{i, j}^{n+1 / 2}\right|\left(1+\tau K\left\|\partial_{u} f\right\|_{\mathbf{L}^{\infty}}\left\|w^{n+1}\right\|_{\mathbf{L}^{\infty}}\right) \\
& +\tau K\left\|\partial_{u} f\right\|_{\mathbf{L}^{\infty}}\left\|w^{n+1}\right\|_{\mathbf{L}^{\infty}} \sum_{i, j} h\left|U_{i, j+1}^{n+1 / 2}-U_{i, j}^{n+1 / 2}\right| \\
& +2 \tau\left\|\partial_{u} f\right\|_{\mathbf{L}^{\infty}} C\left(\left\|w^{n+1}\right\|_{\mathbf{L}^{\infty}}\right)\left\|u^{n}\right\|_{\mathbf{L}^{1}} .
\end{aligned}
$$

By (3.15) and (3.16) we have therefore

$$
\begin{aligned}
\operatorname{TV}\left(U^{n+1}\right) \leq & \left(1+3 \tau K\left\|\partial_{u} f\right\|_{\mathbf{L}^{\infty}}\left\|w^{n+1}\right\|_{\mathbf{L}^{\infty}}\right) \operatorname{TV}\left(U^{n+1 / 2}\right) \\
& +3 \tau\left\|\partial_{u} f\right\|_{\mathbf{L}^{\infty}} C\left(\left\|w^{n+1}\right\|_{\mathbf{L}^{\infty}}\right)\left\|u^{n}\right\|_{\mathbf{L}^{1}} .
\end{aligned}
$$

Analogously to the estimate (3.17) for $\operatorname{TV}\left(U^{n+1}\right)$, we obtain

$$
\begin{aligned}
\operatorname{TV}\left(U^{n+1 / 2}\right) \leq & \left(1+3 \tau K\left\|\partial_{u} f\right\|_{\mathbf{L}^{\infty}}\left\|w^{n+1}\right\|_{\mathbf{L}^{\infty}}\right) \operatorname{TV}\left(u^{n}\right) \\
& +3 \tau\left\|\partial_{u} f\right\|_{\mathbf{L}^{\infty}} C\left(\left\|w^{n+1}\right\|_{\mathbf{L}^{\infty}}\right)\left\|u^{n}\right\|_{\mathbf{L}^{1}}
\end{aligned}
$$

Then,

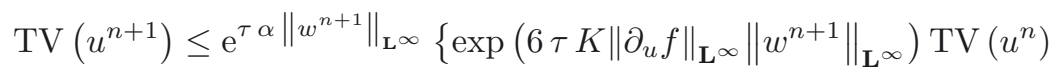

$$
\begin{aligned}
& +\tau \alpha \mathrm{e}^{\tau \beta}\left\|U^{n+1}\right\|_{\mathbf{L}^{\infty}} \mathrm{TV}\left(w^{n+1}\right) \\
& \left.+3 \tau\left\|\partial_{u} f\right\|_{\mathbf{L}^{\infty}} C\left(\left\|w^{n+1}\right\|_{\mathbf{L}^{\infty}}\right)\left\|u^{n}\right\|_{\mathbf{L}^{1}}\left[1+\exp \left(3 \tau K\left\|\partial_{u} f\right\|_{\mathbf{L}^{\infty}}\left\|w^{n+1}\right\|_{\mathbf{L}^{\infty}}\right)\right]\right\} .
\end{aligned}
$$

Collecting the estimates (3.19) and (3.7) of TV $\left(u^{n+1}\right)$ and TV $\left(w^{n+1}\right)$, we obtain now

$$
\begin{aligned}
& \operatorname{TV}\left(u^{n+1}\right)+\operatorname{TV}\left(w^{n+1}\right)
\end{aligned}
$$

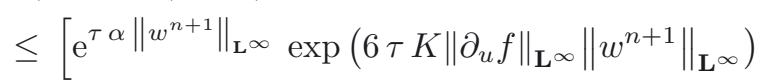

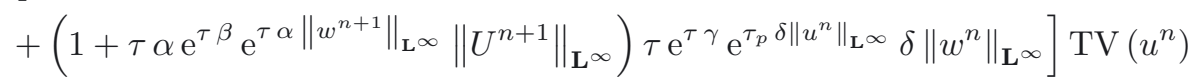

$$
\begin{aligned}
& +\mathrm{e}^{\tau \gamma}\left(1+\tau \alpha \mathrm{e}^{\tau \beta} \mathrm{e}^{\tau \alpha \| w^{n+1}}\left\|_{\mathbf{L}^{\infty}}\right\| U^{n+1} \|_{\mathbf{L}^{\infty}}\right) \operatorname{TV}\left(w^{n}\right)
\end{aligned}
$$

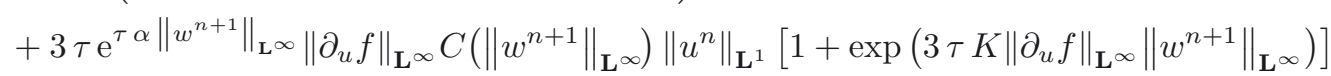

$$
\begin{aligned}
& \leq \mathrm{e}^{\tau K_{1}} \mathrm{TV}\left(u^{n}\right)+\mathrm{e}^{\tau K_{2}} \mathrm{TV}\left(w^{n}\right)+\tau K_{3} \mathrm{e}^{\tau K_{4}},
\end{aligned}
$$

where $K_{l}, l=1, \ldots, 4$ are bounded functions depending on various norm of $u^{n}, w^{n+1}$ and $\partial_{u} f$ as well as on all constants $\alpha, \beta, \gamma, \delta$ and $K$, defined in $(\mathbf{v})$. Defining $\mathcal{K}_{3}=\max \left\{K_{1}, K_{2}\right\}, \mathcal{K}_{4}:=K_{3}$ and $\mathcal{K}_{5}:=K_{4}$ and using induction over $n$ yields now

$$
\mathrm{TV}\left(u^{n}\right)+\mathrm{TV}\left(w^{n}\right) \leq \mathrm{e}^{n \tau \mathcal{K}_{3}}\left[\mathrm{TV}\left(u^{o}\right)+\mathrm{TV}\left(w^{o}\right)+\frac{\mathcal{K}_{4}}{\mathcal{K}_{3}} \mathrm{e}^{\tau \mathcal{K}_{5}}\right]
$$

This completes the proof.

\subsection{Lipschitz continuity in time}

Lemma 3.7. Let assumptions (f), (v) and (0) hold. Then for all $n$ the approximate solution $\left(u^{n}, w^{n}\right)$ constructed by Algorithm 2.1 is such that, for any $n_{1}, n_{2} \in \mathbb{N}$ with $n_{1} \tau \leq T$ and $n_{2} \tau \leq T$,

$$
\left\|u^{n_{1}}-u^{n_{2}}\right\|_{\mathbf{L}^{1}} \leq\left|n_{1}-n_{2}\right| \tau \mathcal{K}_{6}(T, \tau),
$$


where the function $\mathcal{K}_{6}(T, \tau)$ is uniformly bounded for all $n \leq \max \left\{n_{1}, n_{2}\right\}$ and depends on $\alpha, \beta, \gamma, \delta, K$, on various norms of $u, w, \partial_{u} f$, on the total variation of the initial datum and on the map $C$ defined in (v).

Proof. Due to Lemma 3.4, $u^{n}$ is uniformly bounded by some constant depending on $T$. Assumptions (f) and (v) guarantee therefore the Lipschitz continuity of the numerical flux function $F$ defined in (2.4d). Using (2.4e), (2.4f) and (3.18), we can thus conclude

$$
\begin{aligned}
\left\|U^{n+1}-u^{n}\right\|_{\mathbf{L}^{1}} \leq & \sum_{i, j} h^{2}\left(\left|U_{i, j}^{n+1}-U_{i, j}^{n+1 / 2}\right|+\left|U_{i, j}^{n+1 / 2}-u_{i, j}^{n}\right|\right) \\
\leq & \tau \sum_{i, j} h\left[\left|F\left(U_{i, j+1}^{n+1 / 2}, U_{i, j}^{n+1 / 2},(n+1) \tau, x_{i, j+1 / 2}\right)-F\left(U_{i, j}^{n+1 / 2}, U_{i, j-1}^{n+1 / 2},(n+1) \tau, x_{i, j-1 / 2}\right)\right|\right. \\
& \left.+\left|F\left(u_{i+1, j}^{n}, u_{i, j}^{n},(n+1) \tau, x_{i+1 / 2, j}\right)-F\left(u_{i, j}^{n}, u_{i-1, j}^{n},(n+1) \tau, x_{i-1 / 2, j}\right)\right|\right] \\
\leq & \tau \cdot 2 L \sum_{i, j} h\left(\left|U_{i, j+1}^{n+1 / 2}-U_{i, j}^{n+1 / 2}\right|+\left|u_{i+1, j}^{n}-u_{i, j}^{n}\right|\right. \\
& \left.+\left|v_{i+1 / 2, j}^{n+1}-v_{i-1 / 2, j}^{n+1}\right|+\left|v_{i, j+1 / 2}^{n+1}-v_{i, j-1 / 2}^{n+1}\right|\right) \\
\leq & \tau \cdot 2 L\left(\left(2+3 \tau K\left\|\partial_{u} f\right\|_{\mathbf{L}^{\infty}}\left\|w^{n+1}\right\|_{\mathbf{L}^{\infty}}\right) \mathrm{TV}\left(u^{n}\right)+\left\|\nabla v^{n+1}\right\|_{\mathbf{L}^{1}}\right. \\
& \left.+3 \tau\left\|\partial_{u} f\right\|_{\mathbf{L}^{\infty}} C\left(\left\|w^{n+1}\right\|_{\mathbf{L}^{\infty}}\right)\left\|u^{n^{n}}\right\|_{\mathbf{L}^{1}}\right),
\end{aligned}
$$

where $L$ denotes the Lipschitz constant of $F$. Including the source term and defining $T$ such that $\max \left\{n_{1}, n_{2}\right\} \tau \leq$ $T<\infty$, we obtain by $(2.4 \mathrm{~g})$

$$
\begin{aligned}
\left\|u^{n+1}-u^{n}\right\|_{\mathbf{L}^{1}} & \leq \sum_{i, j} h^{2}\left|U_{i, j}^{n+1}-u_{i, j}^{n}\right|+\tau \sum_{i, j} h^{2}\left|U_{i, j}^{n+1}\right|\left|\left(\alpha w_{i, j}^{n+1}-\beta\right)\left(1+\frac{\tau}{2}\left(\alpha w_{i, j}^{n+1}-\beta\right)\right)\right| \\
& \leq\left\|U^{n+1}-u^{n}\right\|_{\mathbf{L}^{1}}+\tau \alpha\left\|U^{n+1}\right\|_{\mathbf{L}^{1}}\left\|w^{n+1}\right\|_{\mathbf{L}^{\infty}} \mathrm{e}^{\tau \alpha\left\|w^{n+1}\right\|_{\mathbf{L}^{\infty}}} \\
& \leq \tau \mathcal{K}_{6}(T, \tau),
\end{aligned}
$$

where $\mathcal{K}_{6}$ is uniformly bounded for all $n \leq \max \left\{n_{1}, n_{2}\right\}$ and all finite $\tau$.

Remark 3.8. Using more refined estimates, the $\mathbf{L}^{1}$ bound for $\nabla v$, necessary in the proof above, can be substituted by the $\mathbf{L}^{\infty}$ bound on $\nabla v$ widely used in the proofs of Lemmas 3.3-3.6. This allows to skip the assumption of $\nabla v$ being bounded in $\mathbf{L}^{1}$ in $(\mathbf{v})$.

\section{Convergence}

For each mesh width $h$, we define $N_{\tau}:=\lfloor T / \tau\rfloor$ and

$$
u_{h}=\sum_{n=0}^{N_{\tau}} \sum_{i, j} u_{i, j}^{n} \chi_{i, j}^{n}, \quad w_{h}=\sum_{n=0}^{N_{\tau}} \sum_{i, j} w_{i, j}^{n} \chi_{i, j}^{n},
$$

where $\chi_{i, j}^{n}$ is the characteristic function of $I_{i, j} \times\left[n \tau,(n+1) \tau\left[\right.\right.$, respectively $I_{i, j} \times\left[N_{\tau} \tau, T\right]$ for the last time step, with $I_{i, j}$ defined as in (2.3).

Theorem 4.1. Let assumptions (f), (v) and (0) hold and fix $0 \leq T<\infty$. Let $h_{\ell}$ be a sequence of grid sizes such that $\lim _{\ell \rightarrow \infty} h_{\ell}=0$ and fix $\lambda>0$ such that the sequence $\tau_{\ell}:=\lambda h_{\ell}$ fulfills (2.1) for all $\ell$. Let $u_{h_{\ell}}$ and $w_{h_{\ell}}$ be given as in (4.1). Then the sequence $\left(u_{h_{\ell}}, w_{h_{\ell}}\right)$ converges to the unique weak solution $(u, w)$ of (1.1). More precisely, $\left(u_{h_{\ell}}\right)$ converges in $\mathbf{L}_{\text {loc }}^{1}$, while $\left(w_{h_{\ell}}\right)$ converges weakly* in $\mathbf{L}^{\infty}$. 
Proof. Thanks to Lemma 3.4

$$
\begin{aligned}
\left\|w_{h}\right\|_{\mathbf{L}^{\infty}\left([0, T] \times \mathbb{R}^{2} ; \mathbb{R}\right)} & \leq \mathrm{e}^{T \gamma}\left\|w^{o}\right\|_{\mathbf{L}^{\infty}\left(\mathbb{R}^{2} ; \mathbb{R}\right)} \\
\left\|u_{h}\right\|_{\mathbf{L}^{\infty}\left([0, T] \times \mathbb{R}^{2} ; \mathbb{R}\right)} & \leq\left\|u^{o}\right\|_{\mathbf{L}^{\infty}\left(\mathbb{R}^{2} ; \mathbb{R}\right)} \exp \left(\left(2 \mathcal{K}_{1}+\mathcal{K}_{2}\right) \frac{1}{\gamma} \mathrm{e}^{T \gamma}\right),
\end{aligned}
$$

so that the sequence $\left(u_{h}, w_{h}\right)$ is bounded in $\mathbf{L}^{\infty}\left([0, T] \times \mathbb{R}^{2} ; \mathbb{R}^{2}\right)$. This implies the existence of a subsequence $\left(u_{h_{k}}, w_{h_{k}}\right)$ that converges weakly* in $\mathbf{L}^{\infty}\left([0, T] \times \mathbb{R}^{2} ; \mathbb{R}^{2}\right)$ to $(u, w)$. Thanks to Lemma $3.5,\left(u_{h_{k}}\right)$ is also uniformly bounded in $\mathbf{L}^{1}\left([0, T] \times \mathbb{R}^{2} ; \mathbb{R}\right)$.

Furthermore, Lemma 3.6 and Lemma 3.7 yield a uniform bound for the space-time total variation of $u_{h_{k}}$, defined by

$$
\operatorname{TV}_{T}\left(u_{h_{k}}\right)=\sum_{n=0}^{N_{\tau}}\left[\tau \operatorname{TV}\left(u_{h_{k}}^{n}\right)+\left\|u_{h_{k}}^{n+1}-u_{h_{k}}^{n}\right\|_{\mathbf{L}^{1}\left(\mathbb{R}^{2} ; \mathbb{R}\right)}\right]
$$

We can thus apply ([10], Thm. 1.7.3) and deduce the existence of $\bar{u} \in \mathbf{B V}_{\text {loc }}\left([0, T] \times \mathbb{R}^{2} ; \mathbb{R}\right)$ and a subsequence of $\left(u_{h_{k}}\right)$ (still denoted by $\left.\left(u_{h_{k}}\right)\right)$ such that

$$
\begin{aligned}
& u_{h_{k}} \rightarrow \bar{u} \text { in } \quad \mathbf{L}_{\text {loc }}^{1}\left([0, T] \times \mathbb{R}^{2} ; \mathbb{R}\right), \\
& u_{h_{k}}(t, x, y) \rightarrow \bar{u}(t, x, y) \quad \text { for a.e. }(t, x, y) \in[0, T] \times \mathbb{R}^{2} .
\end{aligned}
$$

Due to the uniqueness of the limit $\bar{u}$ in $\mathbf{L}^{1}$, shown in [8] (see also Rem. 1.1), we can conclude the convergence of the whole sequence $\left(u_{h_{k}}\right)$ to $\bar{u}$.

From (4.3), it follows easily that $u_{h_{k}}$ converges to $\bar{u}$ also in $\mathbf{L}^{\infty}\left([0, T] \times \mathbb{R}^{2} ; \mathbb{R}\right)$. Since strong convergence implies weak* convergence, we obtain that $u_{h_{k}} \stackrel{*}{\rightarrow} \bar{u}$ in $\mathbf{L}^{\infty}\left([0, T] \times \mathbb{R}^{2} ; \mathbb{R}\right)$. Due to the uniqueness of the weak ${ }^{*}$ limit, we have that $u=\bar{u}$.

By (f), the continuity of the function $f$ implies now that

$$
f\left(u_{h}\right) \rightarrow f(u) .
$$

Note that Lemma 3.4 yields also

$$
\left\|w_{h}(t, \cdot, \cdot)\right\|_{\mathbf{L}^{\infty}\left(\mathbb{R}^{2} ; \mathbb{R}\right)} \leq \mathrm{e}^{T \gamma}\left\|w^{o}\right\|_{\mathbf{L}^{\infty}\left(\mathbb{R}^{2} ; \mathbb{R}\right)} \quad \text { for a.e. } \quad t \in[0, T] .
$$

As above we can thus find a subsequence that converges weakly* in $\mathbf{L}^{\infty}\left(\mathbb{R}^{2} ; \mathbb{R}\right)$ for a.e. $t \in[0, T]$ and due to the uniqueness of the weak* limit, we have

$$
w_{h_{k}}(t, \cdot, \cdot) \stackrel{*}{\rightarrow} w(t, \cdot, \cdot)
$$

Recalling that $\eta \in \mathbf{L}^{1}\left(\mathbb{R}^{2} ; \mathbb{R}\right)$, it is now easy to prove that $\left(w_{h_{k}} * \eta\right)(t, \cdot, \cdot)$ converges (strongly) to $(w * \eta)(t, \cdot, \cdot)$ in $\mathbf{L}^{1}\left(\mathbb{R}^{2} ; \mathbb{R}\right)$ for a.e. $t \in[0, T]$. By $(\mathbf{v})$, and in particular thanks to the fact that the Lipschitz constant of $v$ is bounded, we obtain

$$
v\left(w_{h_{k}} * \eta\right) \rightarrow v(w * \eta) \quad \text { in } \mathbf{L}^{1}\left(\mathbb{R}^{2} ; \mathbb{R}\right) \quad \text { for a.e. } t \in[0, T] .
$$

To prove that $(u, w)$ are weak solutions of $(1.1)$, we choose test functions $\psi \in \mathbf{C}_{c}^{1}\left([0, T], \mathbf{C}_{c}^{2}\left(\mathbb{R}^{2} ; \mathbb{R}\right)\right)$ and $\varphi \in \mathbf{C}_{c}^{1}\left([0, T] \times \mathbb{R}^{2} ; \mathbb{R}\right)$. Define now $\psi_{i, j}^{n, l}:=\psi\left(t^{n, l}, x_{i, j}\right)$, where $t^{n, l}=n \tau+l \tau_{p}$, and

$$
\begin{aligned}
& \psi_{h}=\sum_{n=0}^{N-1} \sum_{i, j} \sum_{l=0}^{m-1} \psi_{i, j}^{n, l} \chi_{i, j}^{n, l} \\
& \delta_{t} \psi_{h}=\sum_{n=0}^{N-1} \sum_{i, j} \sum_{l=0}^{m-1} \frac{\psi_{i, j}^{n, l}-\psi_{i, j}^{n, l-1}}{\tau_{p}} \chi_{i, j}^{n, l} \\
& \Delta_{h} \psi_{h}=\sum_{n=0}^{N-1} \sum_{i, j} \sum_{l=0}^{m-1} \frac{1}{h^{2}}\left(\psi_{i+1, j}^{n, l}+\psi_{i-1, j}^{n, l}+\psi_{i, j+1}^{n, l}+\psi_{i, j-1}^{n, l}-4 \psi_{i, j}^{n, l}\right) \chi_{i, j}^{n, l} .
\end{aligned}
$$


Here, $\chi_{i, j}^{n, l}$ is the characteristic function of $I_{i, j} \times\left[t^{n, l}, t^{n, l+1}\right.$ [, with $I_{i, j}$ defined as in (2.3). Note that $\delta_{t} \psi_{h}$ and $\Delta_{h} \psi_{h}$ are discrete versions of time derivative and Laplace operator. Due to the definition of $\psi_{h}$ and its discrete derivatives, we have strong convergence in $\mathbf{L}^{\infty}\left([0, T] \times \mathbb{R}^{2} ; \mathbb{R}\right)$ for $\psi_{h} \rightarrow \psi$ as well as for the derivatives $\delta_{t} \psi_{h} \rightarrow \partial_{t} \psi$ and $\Delta_{h} \psi_{h} \rightarrow \Delta \psi$ as $h \rightarrow 0$.

Multiply (2.4b) by $h^{2} \psi_{i, j}^{n, l}$ and sum over $n, i, j$ and $l$ to obtain

$$
\begin{aligned}
0= & \tau_{p} h^{2} \sum_{n=0}^{N-1} \sum_{i, j} \sum_{l=0}^{m-1} W_{i, j}^{n, l}\left(\frac{\psi_{i, j}^{n, l}-\psi_{i, j}^{n, l-1}}{\tau_{p}}+\mu \frac{\psi_{i+1, j}^{n, l}+\psi_{i-1, j}^{n, l}+\psi_{i, j+1}^{n, l}+\psi_{i, j-1}^{n, l}-4 \psi_{i, j}^{n, l}}{h^{2}}\right) \\
& +\tau_{p} h^{2} \sum_{n=0}^{N-1} \sum_{i, j} \sum_{l=0}^{m-1} \psi_{i, j}^{n, l}\left(\gamma-\delta u_{i, j}^{n}\right)\left[1+\frac{\tau_{p}}{2}\left(\gamma-\delta u_{i, j}^{n}\right)\right] \frac{W_{i+1, j}^{n, l}+W_{i-1, j}^{n, l}+W_{i, j+1}^{n, l}+W_{i, j-1}^{n, l}}{4} .
\end{aligned}
$$

Using the above convergence results, we can conclude

$$
\int_{0}^{T} \int_{\mathbb{R}^{2}} w \partial_{t} \psi+\mu w \Delta \psi+w(\gamma-\delta u) \psi \mathrm{d} x \mathrm{~d} y \mathrm{~d} t=0
$$

Analogously as above we define

$$
\begin{aligned}
\varphi_{h} & =\sum_{n=0}^{N-1} \sum_{i, j} \varphi_{i, j}^{n} \chi_{i, j}^{n} & \delta_{t} \varphi_{h} & =\sum_{n=0}^{N-1} \sum_{i, j} \frac{\varphi_{i, j}^{n}-\varphi_{i, j}^{n-1}}{\tau} \chi_{i, j}^{n} \\
\delta_{x}^{+} \varphi_{h} & =\sum_{n=0}^{N-1} \sum_{i, j} \frac{\varphi_{i+1, j}^{n}-\varphi_{i, j}^{n}}{h} \chi_{i, j}^{n} & \delta_{x}^{-} \varphi_{h} & =\sum_{n=0}^{N-1} \sum_{i, j} \frac{\varphi_{i, j}^{n}-\varphi_{i-1, j}^{n}}{h} \chi_{i, j}^{n} . \\
\delta_{y}^{+} \varphi_{h} & =\sum_{n=0}^{N-1} \sum_{i, j} \frac{\varphi_{i, j+1}^{n}-\varphi_{i, j}^{n}}{h} \chi_{i, j}^{n} & \delta_{y}^{-} \varphi_{h} & =\sum_{n=0}^{N-1} \sum_{i, j} \frac{\varphi_{i, j}^{n}-\varphi_{i, j-1}^{n}}{h} \chi_{i, j}^{n}
\end{aligned}
$$

and recall that we have $\varphi_{h} \rightarrow \varphi$ and $\delta_{\ell}^{ \pm} \varphi_{h} \rightarrow \partial_{\ell} \varphi$ in $\mathbf{L}^{\infty}\left([0, T] \times \mathbb{R}^{2} ; \mathbb{R}\right)$ for $h \rightarrow 0$ and $\ell=t, x, y$. Multiplying (2.4e)-(2.4g) by $h^{2} \varphi_{i, j}^{n}$ and summing over all $n, i$ and $j$ we obtain

$$
\begin{aligned}
0= & \tau h^{2} \sum_{n=0}^{N-1} \sum_{i, j} u_{i, j}^{n} \frac{\varphi_{i, j}^{n}-\varphi_{i, j}^{n-1}}{\tau}+\tau h^{2} \sum_{n=0}^{N-1} \sum_{i, j}\left(\alpha w_{i, j}^{n}-\beta\right) \varphi_{i, j}^{n} U_{i, j}^{n+1} \\
& +\tau h^{2} \sum_{n=0}^{N-1} \sum_{i, j} \frac{1}{2} f\left(u_{i, j}^{n}\right)\left(v_{i-1 / 2, j}^{n+1} \frac{\varphi_{i, j}^{n}-\varphi_{i-1, j}^{n}}{h}+v_{i+1 / 2, j}^{n+1} \frac{\varphi_{i+1, j}^{n}-\varphi_{i, j}^{n}}{h}\right) \\
& +\tau h^{2} \sum_{n=0}^{N-1} \sum_{i, j} \frac{1}{2} f\left(U_{i, j}^{n+1 / 2}\right)\left(v_{i, j-1 / 2}^{n+1} \frac{\varphi_{i, j}^{n}-\varphi_{i, j-1}^{n}}{h}+v_{i, j+1 / 2}^{n+1} \frac{\varphi_{i, j+1}^{n}-\varphi_{i, j}^{n}}{h}\right) \\
& +h^{2} \sum_{n=0}^{N-1} \sum_{i, j} \frac{h^{2}}{4}\left(u_{i, j}^{n} \frac{\varphi_{i-1, j}^{n}-2 \varphi_{i, j}^{n}+\varphi_{i+1, j}^{n}}{h^{2}}+U_{i, j}^{n+1 / 2} \frac{\varphi_{i, j-1}^{n}-2 \varphi_{i, j}^{n}+\varphi_{i, j+1}^{n}}{h^{2}}\right) \\
& +\tau h^{2} \sum_{n=0}^{N-1} \sum_{i, j} \frac{\tau}{2}\left(\alpha w_{i, j}^{n}-\beta\right)^{2} \varphi_{i, j}^{n} U_{i, j}^{n+1} .
\end{aligned}
$$

Recall that $w_{h}$ is uniformly bounded in $\mathbf{L}^{\infty}$ for all $h$. This directly implies that also $w_{h}^{2}$ is uniformly bounded in $\mathbf{L}^{\infty}$ and thus converges weakly* to some function $g \in \mathbf{L}^{\infty}\left([0, T] \times \mathbb{R}^{2} ; \mathbb{R}\right)$. Due to the smoothness of $v$ and 
the convergence of $u_{h}, w_{h}, w_{h}^{2}$ and $\varphi_{h}$, we can thus deduce that the limit functions $u, w$ and $\varphi$ fulfill

$$
\int_{0}^{T} \int_{\mathbb{R}^{2}} u \partial_{t} \varphi+f(u) v \cdot \operatorname{div}(\varphi)+(\alpha w-\beta) \varphi \mathrm{d} x \mathrm{~d} y \mathrm{~d} t=0 .
$$

We proved that $(u, w)$ is a weak solution to (1.1). Since by [8] and Remark 1.1 we know that the weak solution to $(1.1)$ is unique, $(u, w)$ is the unique weak solution.

\section{Numerical EXAMPLES}

To conclude the paper, we present some numerical examples that show on one hand the convergence of the scheme and on the other hand some qualitative properties of the system (1.1). In all examples, we make the following choice for the vector field $v$ :

$$
\boldsymbol{v}(w)=\kappa \frac{\nabla(w * \eta)}{\sqrt{1+\|\nabla(w * \eta)\|^{2}}},
$$

where the compactly supported kernel function $\eta$ is chosen as follows

$$
\eta(x, y)=\hat{\eta}\left(\ell^{2}-\|(x, y)\|^{2}\right)^{3} \chi_{B(0, \ell)} \quad \text { with } \hat{\eta} \in \mathbb{R}^{+} \text {such that } \iint_{\mathbb{R}^{2}} \eta(x, y) \mathrm{d} x \mathrm{~d} y=1
$$

The positive parameter $\ell$ represents the maximal distance at which predators $u$ feel the presence of prey $w$. It can be easily verified that (5.1) fulfills the assumption (v).

We compute the numerical solution on the domain

$$
D=[0,0.5] \times[0,1]
$$

and consider the following sizes of the space mesh:

$$
h=0.005, \quad h=0.0025, \quad h=0.00125 .
$$

Since no exact solutions are available, we use the numerical solution computed for $h=0.000625$ as reference solution $(u, w)$. The corresponding time step sizes $\tau_{p}$ and $\tau$ are chosen according to the definition in Section 2, see especially (2.1) and the lines above.

Let $\left(u_{h}, w_{h}\right)$ be the numerical solution associated to space mesh size $h$. The error is computed in the following way

$$
\begin{aligned}
\left\|u_{h}-u\right\|_{\mathbf{L}^{1}} & =\sup _{t \in[0, T]}\left\|u_{h}(t)-u(t)\right\|_{\mathbf{L}^{1}(D ; \mathbb{R})}, \\
\left\|w_{h}-w\right\|_{\mathbf{L}^{1}} & =\sup _{t \in[0, T]}\left\|w_{h}(t)-w(t)\right\|_{\mathbf{L}^{1}(D ; \mathbb{R})} .
\end{aligned}
$$

More precisely, we average the reference solution $(u, w)$ on the coarse grid in order to compare it to the solution $\left(u_{h}, w_{h}\right)$.

We define $\mathrm{EOC}_{u}$, respectively $\mathrm{EOC}_{w}$, the experimental order of convergence for $u$, respectively for $w$, computed as follows:

$$
\operatorname{EOC}_{u}=\frac{\log \frac{\left\|u_{1}-u\right\|_{\mathbf{L}^{1}}}{\left\|u_{2}-u\right\|_{\mathbf{L}^{1}}}}{\log \frac{h_{1}}{h_{2}}}, \quad \operatorname{EOC}_{w}=\frac{\log \frac{\left\|w_{1}-w\right\|_{\mathbf{L}^{1}}}{\left\|w_{2}-w\right\|_{\mathbf{L}^{1}}}}{\log \frac{h_{1}}{h_{2}}}
$$

where $\left(u_{1}, w_{1}\right),\left(u_{2}, w_{2}\right)$ are solutions with grid size $h_{1}$ and $h_{2}$ respectively. 


\subsection{Example 1}

In our first example, we consider the test case proposed in ([8], Sect. 3.1), where the parameters are chosen as

$$
\begin{array}{llll}
\alpha=2 & \beta=1 & \kappa=1 & \\
\gamma=1 & \delta=2 & \mu=0.5 & \ell=0.0375
\end{array}
$$

with the following initial datum on $D$

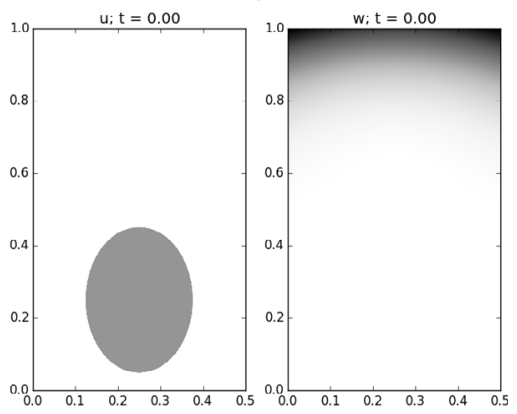

$$
\begin{aligned}
& u_{o}(x, y)=4 \chi_{A}(x, y) \\
& w_{o}(x, y)=3(2 y-1) \max \{0, h(x, y)\} \chi_{B}(x, y)
\end{aligned}
$$

where

$$
\begin{aligned}
& h(x, y)=(4 x-1)^{2}+(4 y-2)^{2}-0.25 \\
& A=\left\{(x, y) \in \mathbb{R}^{2}:(8 x-2)^{2}+(1.25(4 y-1))^{2} \leq 1\right\} \\
& B=\left\{(x, y) \in \mathbb{R}^{2}: y \geq 0.5\right\} .
\end{aligned}
$$

For this example we consider two hyperbolic flux functions

1a. $f(u)=u$, as in $[8]$;

1b. $f(u)= \begin{cases}\frac{u^{2}}{1728}(10-u)^{3} & \text { if } 0 \leq u \leq 10 \\ 0 & \text { elsewhere. }\end{cases}$

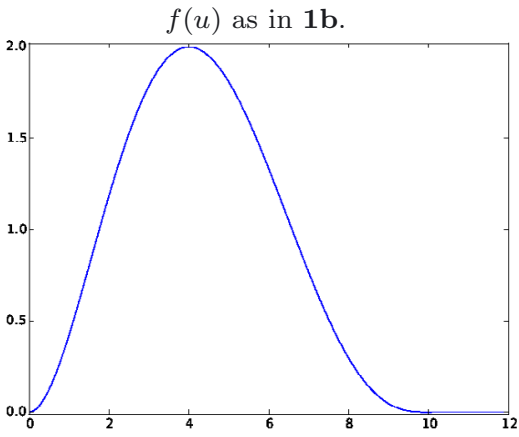

It is easy to see that both functions fulfill assumption (f). The constants in case $1 \mathrm{~b}$ are related to the initial datum. Indeed, this choice guarantees that $f(4)=2$ is the maximal value of $f$. Note that $\left\|\partial_{u} f\right\|_{\mathbf{L}^{\infty}}=$ $5(3+8 \sqrt{6}) / 144 \approx 0.78458 \leq 1$.

To evaluate the convolution without boundary effects, we compute the solution on a slightly bigger domain than $D$. More precisely, we enlarge the computational domain $D$ by adding in all directions a constant quantity $\mathcal{C}(\eta)>0$, related to the size of the support of the kernel function $\eta$. The enlarged domain is thus of the form

$$
[-\mathcal{C}(\eta), 0.5+\mathcal{C}(\eta)] \times[-\mathcal{C}(\eta), 1+\mathcal{C}(\eta)] \supseteq D .
$$

In particular, we choose $C(\eta)$ large enough such that, when computing the convolution for a point on the boundary of $D$, the whole support of the kernel function $\eta$ is inside the extended computational domain.

The boundary conditions for $u$ and $w$ are chosen to remain equal to the initial datum all along the boundary of this extended computational domain. For the balance law, this means to assume a constant value outside the computational domain and to compute the flux accordingly: whenever $\boldsymbol{v}(w)$ is pointing inward the domain, an inflow due to the boundary condition is considered. Concerning the parabolic equation, this choice of boundary conditions amounts to assume that the displayed solution is part of a solution defined on all $\mathbb{R}^{2}$ that gives constant inflow into the computational domain.

The solution is computed up to time $T_{\max }=0.3$ in Example $1 \mathrm{a}$ and up to time $T_{\max }=0.5$ in Example $1 \mathrm{~b}$. 
TABLE 1. $\mathbf{L}^{1}$-error computed as in (5.3) and experimental order of convergence computed as in (5.4) for the solution to (1.1), (5.1) and (5.5) with initial datum (5.6) and flux function $f$ as in $1 \mathrm{a}$.

\begin{tabular}{lcccc}
\hline $\boldsymbol{h}$ & $\left\|\boldsymbol{u}_{\boldsymbol{h}}-\boldsymbol{u}\right\|_{\mathrm{L}^{1}}$ & $\mathrm{EOC}_{u}$ & $\left\|\boldsymbol{w}_{\boldsymbol{h}}-\boldsymbol{w}\right\|_{\mathrm{L}^{1}}$ & $\mathrm{EOC}_{\boldsymbol{w}}$ \\
\hline 0.005 & 0.56 & - & 0.303 & - \\
0.0025 & 0.275 & 1.03 & 0.114 & 1.41 \\
0.00125 & 0.106 & 1.38 & 0.034 & 1.74 \\
\hline
\end{tabular}

TABLE 2. $\mathbf{L}^{1}$-error computed as in (5.3) and experimental order of convergence computed as in (5.4) for the solution to (1.1), (5.1) and (5.5) with initial datum (5.6) and flux function $f$ as in $1 \mathrm{~b}$.

\begin{tabular}{lcccc}
\hline $\boldsymbol{h}$ & $\left\|\boldsymbol{u}_{\boldsymbol{h}}-\boldsymbol{u}\right\|_{\mathbf{L}^{1}}$ & $\mathrm{EOC}_{u}$ & $\left\|\boldsymbol{w}_{\boldsymbol{h}}-\boldsymbol{w}\right\|_{\mathbf{L}^{1}}$ & $\mathrm{EOC}_{\boldsymbol{w}}$ \\
\hline 0.005 & 0.579 & - & 0.43 & - \\
0.0025 & 0.273 & 1.08 & 0.185 & 1.22 \\
0.00125 & 0.101 & 1.43 & 0.064 & 1.54 \\
\hline
\end{tabular}

TABLE 3. Space-time minimum values of $u_{h}$ and $w_{h}$ for the solution to (1.1), (5.1) and (5.5) with initial datum (5.6) and flux function $f$ as in $1 \mathrm{a}$ (left) and $1 \mathrm{~b}$ (right).

\begin{tabular}{lcc}
\hline$h$ & $\min _{i, j, n} u_{h}$ & $\min _{i, j, n} w_{h}$ \\
\hline 0.005 & 0 & 0 \\
0.0025 & 0 & 0 \\
0.00125 & 0 & 0 \\
\hline
\end{tabular}

\begin{tabular}{lcc}
\hline$h$ & $\min _{i, j, n} u_{h}$ & $\min _{i, j, n} w_{h}$ \\
\hline 0.005 & 0 & 0 \\
0.0025 & 0 & 0 \\
0.00125 & 0 & 0 \\
\hline
\end{tabular}

In Tables 1 and 2 we report the values of the $\mathbf{L}^{1}$-error for the different mesh sizes and the corresponding experimental order of convergence for flux function $1 \mathrm{a}$ and $1 \mathrm{~b}$ respectively. Figure 1 displays the error in logarithmic scale. The lines obtained connecting the values for $u$, respectively $w$, can be easily compared with the line with slope 1 , that represents the order of convergence we expect theoretically for smooth solutions.

To emphasize that the method is indeed positivity preserving as stated in the analytical part, we report the space-time minimum values of $u_{h}$ and $w_{h}$ in Table 3 .

Figures 2 and 3 show the results of the numerical integration for flux function $f$ as in $1 \mathrm{a}$ and $1 \mathrm{~b}$ respectively. The figures illustrate well also the spatial effect of the model: thanks to the non-locality of $\boldsymbol{v}$, predators $u$ move towards regions where the concentration of prey $w$ is higher. Observe that boundary effects are negligible at least up to the maximal time of integration.

\subsection{Example 2}

In this example, we modify the treatment of the boundary. In particular, we impose isolating boundary conditions for $u$ and Neumann homogeneous boundary conditions for $w$. We consider

$$
f(u)=u(1-u),
$$



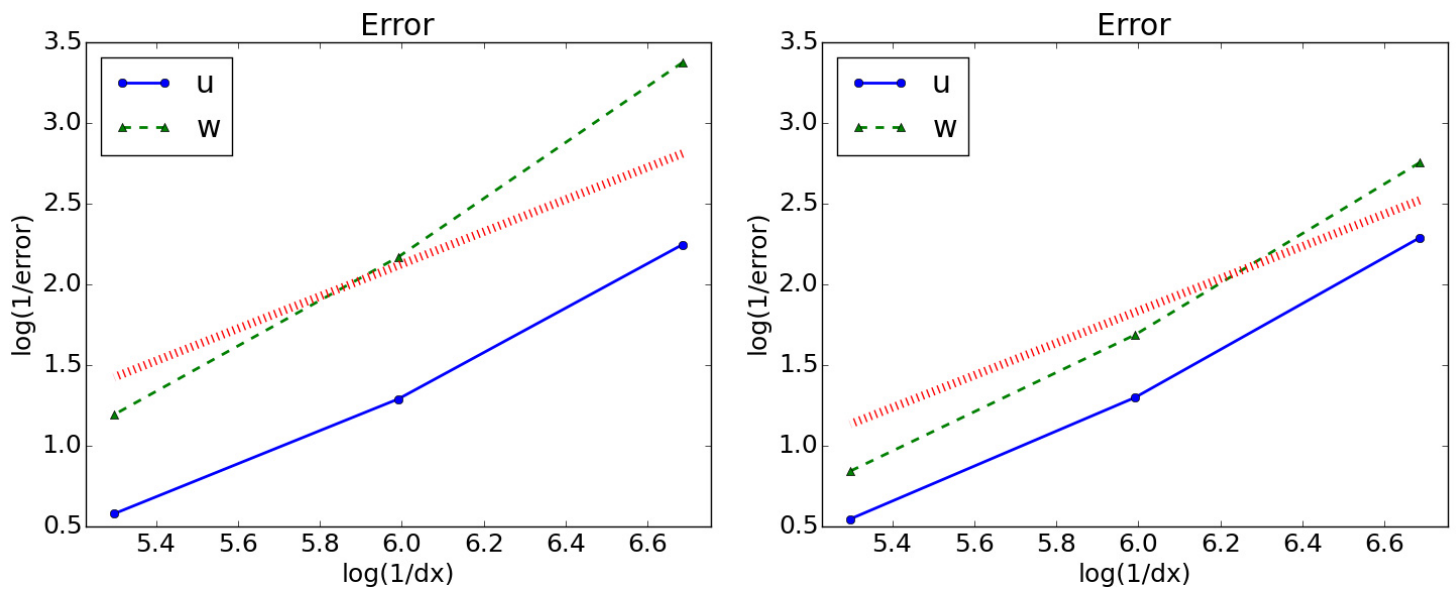

Figure 1. Plot of the $\mathbf{L}^{1}$-error the solution to (1.1), (5.1) and (5.5) with initial datum (5.6): case $1 \mathrm{a}$ on the left, case $1 \mathrm{~b}$ on the right. The dotted line has slope 1 and represents the order of convergence we expect theoretically.
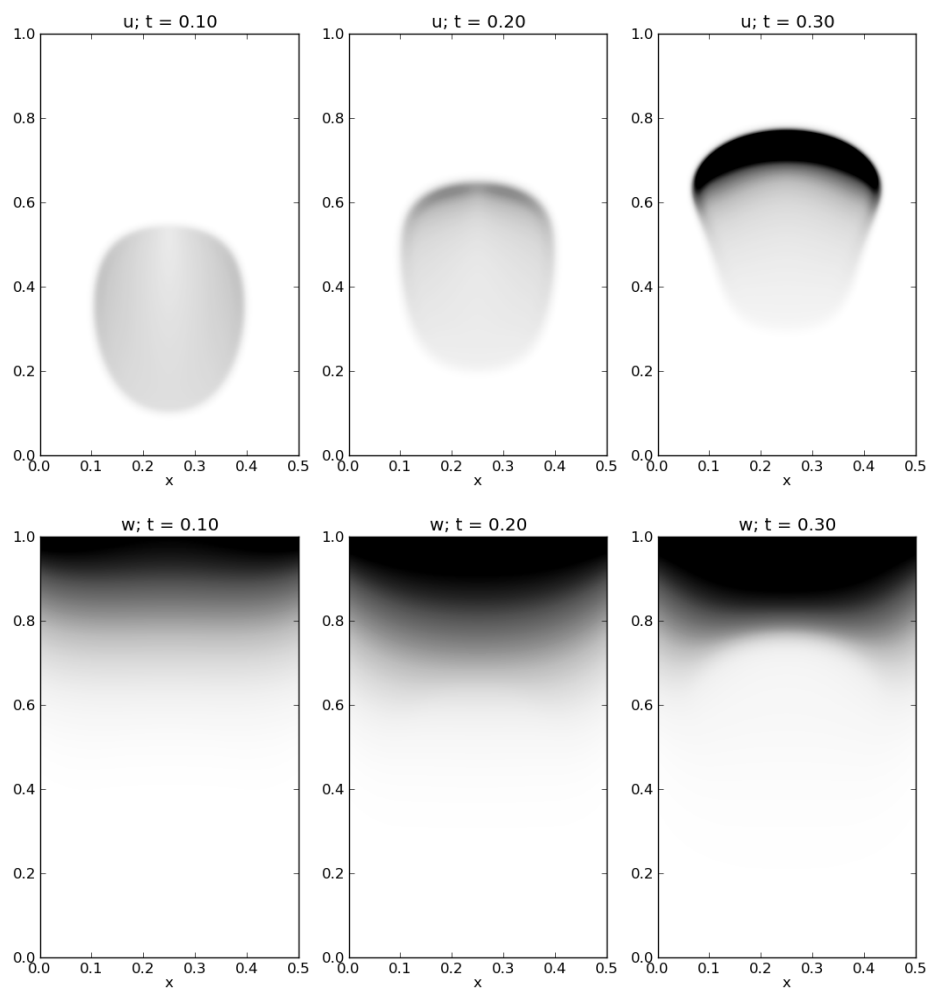

Figure 2. Numerical integration of (1.1), (5.1) and (5.5) with initial datum (5.6) and $f$ as in 1 a at time $t=0.1,0.2,0.3$. This solution was obtained with $h=0.000625$. Top row: $u$, bottom row: $w$. Darker colors indicate higher densities. 

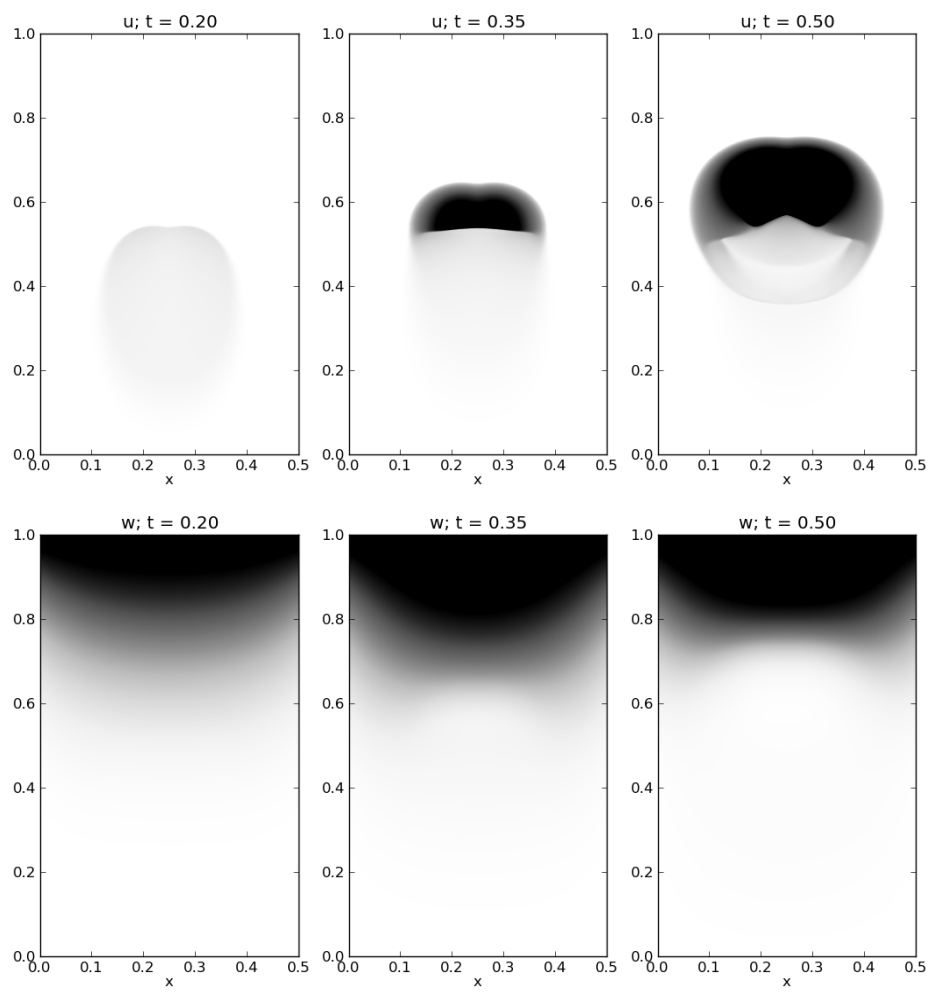

FigURE 3. Numerical integration of (1.1), (5.1) and (5.5) with initial datum (5.6) and $f$ as in $1 \mathrm{~b}$ at time $t=0.2,0.35,0.5$. This solution was obtained with $h=0.000625$. Top row: $u$, bottom row: $w$. Darker colors indicate higher densities.

and it is easy to see that it fulfills the assumption (f). We set

$$
\begin{array}{llll}
\alpha=2 & \beta=0.8 & \kappa=1 & \\
\gamma=0.8 & \delta=24 & \mu=0.1 \quad \ell=0.0625
\end{array}
$$

with the following initial datum on $D$
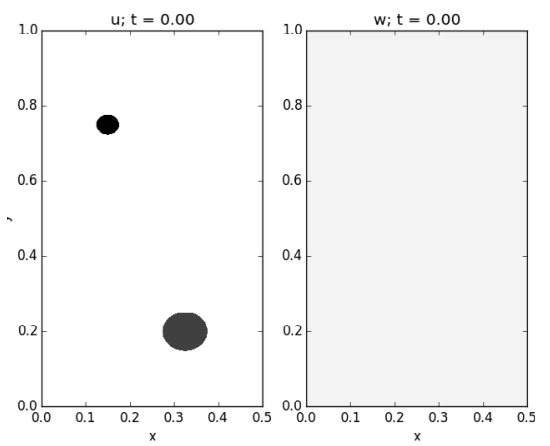

$$
\begin{aligned}
u_{o}(x, y) & =0.05\left(5 \chi_{E}(x, y)+4 \chi_{F}(x, y)\right) \\
w_{o}(x, y) & =0.2
\end{aligned}
$$

where

The solution is computed up to time $T_{\max }=4$ on a mesh of width $h=0.00125$. Figure 4 shows the evolution of the total mass (i.e. the $\mathbf{L}^{1}$-norm in space) of predators $u$ and preys $w$ over time, while Figure 5 shows the spacial 

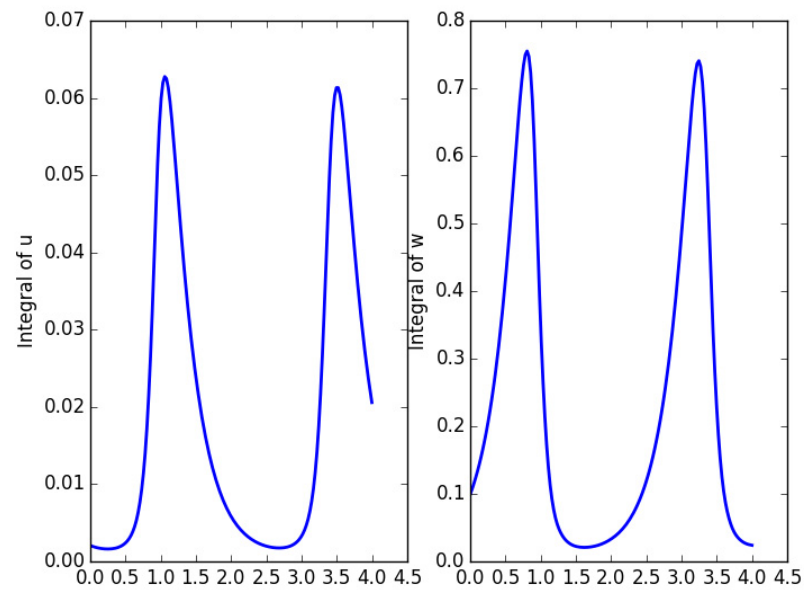

Figure 4. The typical Lotka-Volterra effect obtained for the solution to (1.1), (5.1) and (5.7) and (5.8) with initial datum (5.9) and a mesh of width $h=0.00125$. The graphs display the integral of $u$ (left), respectively $w$ (right), representing the total mass of predators and prey.
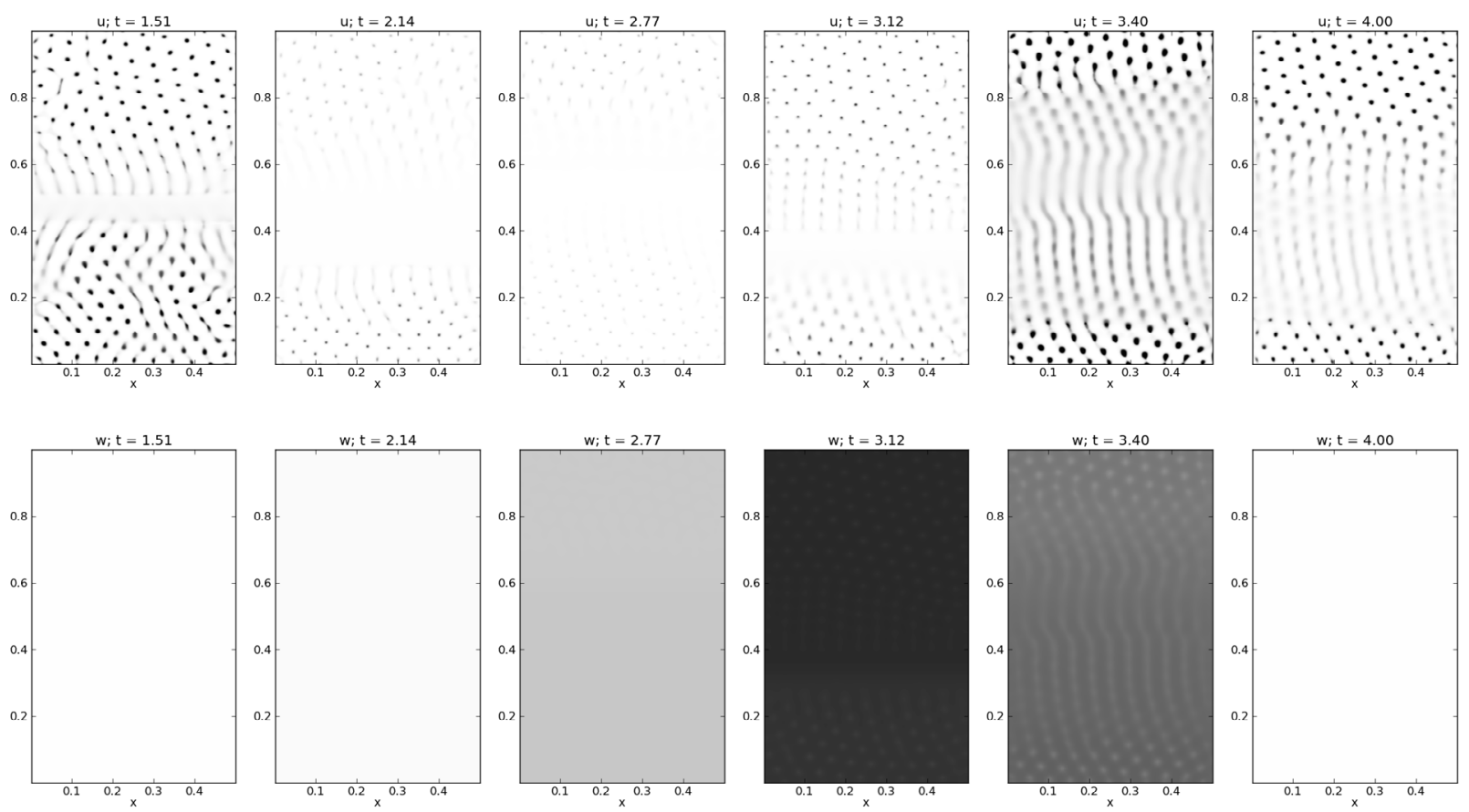

Figure 5. Numerical integration of (1.1), (5.1), (5.7) and (5.8) with initial datum (5.9) and a mesh of width $h=0.00125$. Top row: $u$, bottom row: $w$. Darker colors indicate higher densities. The first column shows the spacial distribution of $u$ and $w$ at a time where $\|w\|_{\mathbf{L}^{1}}$ attains its minimum, while $\|u\|_{\mathbf{L}^{1}}$ is already decreasing. The second column represents some intermediate time, where $\|u\|_{\mathbf{L}^{1}}$ is still decreasing while $\|w\|_{\mathbf{L}^{1}}$ has already started to increase. The third column shows a scenario where $\|u\|_{\mathbf{L}^{1}}$ is minimal, while the forth column shows $u$ and $w$ when $\|w\|_{\mathbf{L}^{1}}$ is maximal. In the fifth column, $\|u\|_{\mathbf{L}^{1}}$ is maximal, while $\|w\|_{\mathbf{L}^{1}}$ has started to decrease again. Finally, in the last column, $\|w\|_{\mathbf{L}^{1}}$ is again minimal, while $\|u\|_{\mathbf{L}^{1}}$ is still decreasing. 
TABLE 4. Space-time minimum values of $u_{h}$ and $w_{h}$ for the solution to Example 2 using equations (1.1), (5.1), (5.7) and (5.8) and initial datum (5.9).

\begin{tabular}{lcc}
\hline$h$ & $\min _{i, j, n} u_{h}$ & $\min _{i, j, n} w_{h}$ \\
\hline 0.00125 & 0 & 0.04 \\
\hline
\end{tabular}

behaviour of the solution at different times. In this example we can clearly see the typical Lotka-Volterra effect, see Figure 4, where the evolution of the total mass of predators and prey in time is shown. One population, in this case predators $u$, apparently almost disappear, then its mass rises again, due to feeding on prey and to newborns. At the same time the other population grows, until its mass reaches a sort of maximum point: from that instant on, predators eating prey produce a decrease in prey mass. However, when the total mass of prey is very low, predators have nothing left to eat, hence they decrease, while prey are free to increase, and the whole cycle begins again. This time-periodic behaviour can also be seen in the spacial behaviour, see Figure 5 . There, almost the same spacial-patterns for $u$ and $w$ appear in every cycle.

As for the previous examples, we report the space-time minimum values of $u_{h}$ and $w_{h}$ in Table 4 to emphasize that the method is indeed positivity preserving.

\section{REFERENCES}

[1] A. Aggarwal, R.M. Colombo and P. Goatin, Nonlocal systems of conservation laws in several space dimensions. SIAM J. Numer. Anal. 53 (2015) 963-983.

[2] P. Amorim, R.M. Colombo and A. Teixeira, On the numerical integration of scalar nonlocal conservation laws. ESAIM: M2AN 49 (2015) 19-37.

[3] R. Arditi and L. Ginzburg, Coupling in predator-prey dynamics: ratio dependence. J. Theoret. Biol. 139 (1989) $311-326$.

[4] M.S. Bartlett. On theoretical models for competitive and predatory biological systems. Biometrika 44 (1957) $27-42$.

[5] G.I. Bell, Mathematical model of clonal selection and antibody production. J. Theoret. Biol. 29 (1970) $191-232$.

[6] C. Chainais-Hillairet, Finite volume schemes for a nonlinear hyperbolic equation. Convergence towards the entropy solution and error estimate. ESAIM: M2AN 33 (1999) 129-156.

[7] C. Chainais-Hillairet and S. Champier, Finite volume schemes for nonhomogeneous scalar conservation laws: error estimate. Numer. Math. 88 (2001) 607-639.

[8] R.M. Colombo and E. Rossi, Hyperbolic predators vs. parabolic prey. Commun. Math. Sci. 13 (2015) 369-400.

[9] M.G. Crandall and A. Majda, Monotone difference approximations for scalar conservation laws. Math. Comput. 34 (1980) $1-21$.

[10] C.M. Dafermos, Hyperbolic conservation laws in continuum physics. In vol. 325 of Grundlehren der Mathematischen Wissenschaften [Fundamental Principles of Mathematical Sciences]. 2nd edition. Springer-Verlag, Berlin (2005).

[11] R. Eymard, T. Gallouët, M. Ghilani and R. Herbin, Error estimates for the approximate solutions of a nonlinear hyperbolic equation given by finite volume schemes. IMA J. Numer. Anal. 18 (1998) 563-594.

[12] R.M. Goodwin, A Growth Cycle. In Socialism, Capitalism and Economic Growth, edited by C.H. Feinstein. Cambridge University Press (1967) 54-59.

[13] C.S. Holling, The components of predation as revealed by a study of small-mammal predation of the european pine sawfly. The Canadian Entomologist 91 (1959) 293-320.

[14] K.H. Karlsen and J.D. Towers, Convergence of the Lax-Friedrichs scheme and stability for conservation laws with a discontinous space-time dependent flux. Chinese Ann. Math. Ser. B 25 (2004) 287-318.

[15] A.J. Lotka, Elements of Physical Biology. Williams and Wilkins (1925).

[16] G.H. Pimbley, Jr, Periodic solutions of predator-prey equations simulating an immune response. I. Math. Biosci. 20 (1974) $27-51$.

[17] V. Volterra, Variazioni e fluttuazioni del numero d'individui in specie animali conviventi. Mem. Acad. Lincei Roma 2 (1926) 31-113. 